\title{
Inverse Compton emission from relativistic jets in binary systems
}

\author{
Dmitry Khangulyan, ${ }^{1,2 \star}$ Valentí Bosch-Ramon ${ }^{3}$ and Yasunobu Uchiyama ${ }^{1}$ \\ ${ }^{1}$ Department of Physics, Rikkyo University, Nishi-Ikebukuro 3-34-1, Toshima-ku, Tokyo 171-8501, Japan \\ ${ }^{2}$ RIKEN iTHEMS, Hirosawa 2-1, Wako, Saitama 351-0198, Japan \\ ${ }^{3}$ Departament de Física Quàntica i Astrofísica, Institut de Ciències del Cosmos (ICC), Universitat de Barcelona (IEEC-UB), Martí i Franquès 1, E08028 \\ Barcelona, Spain
}

Accepted 2018 August 28. Received 2018 August 20; in original form 2018 July 10

\begin{abstract}
The gamma-ray emission detected from several microquasars can be produced by relativistic electrons emitting through inverse Compton scattering. In particular, the $\mathrm{GeV}$ emission detected from Cygnus X-3, and its orbital phase dependence, strongly suggests that the emitting electrons are accelerated in a relativistic jet, and that the optical companion provides the dominant target. Here, we study the effects related to particle transport in the framework of the relativistic jet scenario. We find that even in the most compact binary systems, with parameters similar to Cygnus X-3, particle transport can have a substantial influence on the GeV light curve unless the jet is slow, $\beta<0.7$. In more extended binary systems, strong impact of particle transport is nearly unavoidable. Thus, even for a very compact system such as Cygnus X-3, particle transport significantly affects the ability of one-zone models to infer the properties of the gamma-ray production site based on the shape of the $\mathrm{GeV}$ lightcurve. We conclude that a detailed study of the gamma-ray spectrum can further constrain the structure and other properties of the gamma-ray emitter in Cygnus X-3, although such a study should account for gamma-gamma attenuation, since it may strongly affect the spectrum above $5 \mathrm{GeV}$.
\end{abstract}

Key words: radiation mechanisms: non-thermal-methods: analytical-binaries: generalgamma-rays: stars.

\section{INTRODUCTION}

Microquasars $(\mu \mathrm{Q})$ are binary systems that host a companion star and an accreting compact object (CO) from which jets are produced. Several $\mu \mathrm{Q}$ have been detected in the GeV gamma-ray range with AGILE and Fermi LAT (Abdo et al. 2009; Tavani et al. 2009; Williams et al. 2011; Bulgarelli et al. 2012; Corbel et al. 2012; Piano et al. 2012; Bodaghee et al. 2013; Malyshev, Zdziarski \& Chernyakova 2013; Zanin et al. 2016; Piano et al. 2017). The variability found in the $\mathrm{GeV}$ emission in some of these sources is consistent with inverse Compton (IC) scattering of stellar photons by relativistic electrons accelerated in the jets (e.g. Dubus, Cerutti \& Henri 2010a; Zanin et al. 2016; Zdziarski et al. 2018, see also Jackson 1972). The IC origin of the gamma-ray emission detected from $\mu \mathrm{Q}$ is supported by arguments based on higher efficiency of leptonic radiation mechanisms, compared to hadronic ones, under conditions of compact binary systems (Bosch-Ramon \& Khangulyan 2009).

If the dominant target photon field is provided by the stellar companion, IC scattering will be strongly anisotropic (see e.g. Khangulyan \& Aharonian 2005; Khangulyan, Aharonian \& Bosch-
Ramon 2008), and the scattering angle will change along the orbit. This variability of the scattering angle is imprinted in the emission intensity and may be the dominant factor shaping the $\mathrm{GeV}$ light curve (e.g. Dubus et al. 2010a, for Cygnus X-3). The specific dependence of the scattering angle on the orbital phase is determined by the jet and counter-jet orientations and the location of the acceleration and the emission sites in the jet. Thus, gamma-ray light curves can help in constraining the emitter location in $\mu \mathrm{Q}$.

Cygnus X-3 is the brightest and best-studied gamma-ray emitting $\mu Q$ (e.g. Abdo et al. 2009; Tavani et al. 2009). The high luminosity of this source may favour, from energetic arguments, relativistic jet velocities, as they could alleviate the demanding energy requirements through Doppler boosting. In such a jet, the non-thermal distribution of particles and their emission would be significantly affected by relativistic effects. Nevertheless, a highly relativistic jet is somewhat in tension with Fermi LAT data in the context of a one-zone IC emitter (Dubus et al. 2010a; Zdziarski et al. 2018). On the other hand, radio VLBI observations of the jets of Cygnus X-3, from milliarcsecond-to-arcsecond scales $\left(\sim 10-10^{4}\right.$ au), favour an at least moderately relativistic jet (Martí, Paredes \& Peracaula 2001; Mioduszewski et al. 2001), which may point to an even more relativistic flow on the scales of the binary $(\sim 0.1 \mathrm{au})$. 
In this paper, we derive the formulas for the IC emission from a relativistic jet using the distribution function of electrons in the phase space $(\boldsymbol{r}, \boldsymbol{p})$, with $\boldsymbol{r}$ and $\boldsymbol{p}$ being the particle spatial and momentum coordinates in the laboratory reference frame (RF), respectively. This function is a Lorentz invariant, which allows us to avoid cumbersome RF transformations in the case when the contribution from synchrotron self-Compton (SSC) is negligible ${ }^{1}$. This approach also allows us to obtain the results in a form that consistently describes the advection and radiation of gamma-rays by particles in the case of an extended emitter (EE). In the derivation, we account both for the transformation of the particle distribution to the laboratory frame and for the impact of relativistic effects on the particle cooling in the plasma frame. We obtain an analytic solution for the invariant distribution function under the assumption of dominant Thomson IC losses and numerically compute the IC radiation accounting for changes in the target density and scattering angle along the jet. We discuss the impact of the synchrotron and adiabatic losses, and characterize the conditions when synchrotron losses dominate, under which an analytic solution for the particle distribution can be obtained.

An approach based on the invariant distribution function was earlier suggested to describe the beaming pattern of the external IC emission produced by blobs moving relativistically in blazar jets (Georganopoulos, Kirk \& Mastichiadis 2001). This approach was later applied to study variable IC emission in binary systems (see e.g. Georganopoulos, Aharonian \& Kirk 2002; Romero, Kaufman Bernadó \& Mirabel 2002; Kaufman Bernadó, Romero \& Mirabel 2002). In contrast to these studies, in our paper, we consider the emission produced in a jet that implies a different beaming pattern compared to an emitting blob. Another difference with the calculations presented by Georganopoulos et al. (2001) is that we use the invariant distribution function to describe the propagation and cooling of relativistic electrons in an $\mathrm{EE}$, which appears to be an important factor for interpreting the gamma-ray emission detected from gamma-ray binary systems.

Compared to other models, which involve EEs in $\mu \mathrm{Q}$ (see e.g. Vila \& Romero 2010; Vila, Romero \& Casco 2012; Zdziarski et al. 2014a,b; Pepe, Vila \& Romero 2015) and rely on the conventional approach with RF transformations, our method significantly simplifies the computation of the external IC emission. Thus, this paper allows us to extend the existing models focusing on the GeV gamma-ray emission from $\mu \mathrm{Q}$ (e.g. Dubus et al. 2010a; Zdziarski et al. 2012, 2018), and to study consistently the influence of particle advection on the gamma-ray spectra and light curves.

Under conditions typical for $\mu \mathrm{Q}$, in the TeV energy band the Klein-Nishina regime and gamma-gamma attenuation can affect the IC scattering and propagation of gamma-rays, respectively (see e.g. Bosch-Ramon \& Khangulyan 2009). In some systems with particularly hot stellar companions, e.g. as Cygnus X-3, these effects may influence the production of $\mathrm{GeV}$ gamma-rays (see e.g. Protheroe \& Stanev 1987; Moskalenko, Karakula \& Tkaczyk 1993; Bednarek 1997; Cerutti et al. 2011; Sitarek \& Bednarek 2012). Therefore, we also consider the influence of the Klein-Nishina effect on the electron transport and the impact of the gamma-gamma absorption on the spectrum adopting system parameters similar to Cygnus X-3.

\footnotetext{
${ }^{1}$ Note that in the case of very clumpy jets, the SSC mechanism may provide a non-negligible contribution (see Zdziarski et al. 2017, for the case of Cygnus X-1)
}

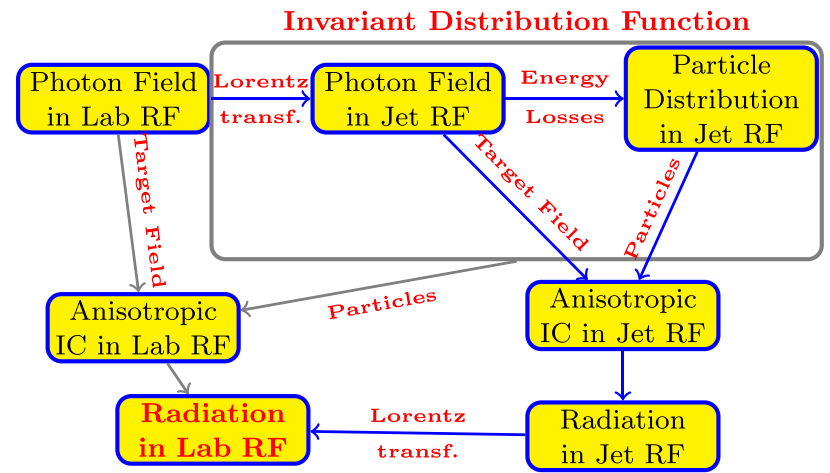

Figure 1. Algorithm for calculating the IC emission using the conventional approach and the Lorentz invariant distribution function.

\section{UPSCATTERING OF EXTERNAL PHOTONS BY A RELATIVISTIC JET}

Conventionally, the IC radiation from relativistic sources is computed as follows. First, one transforms the radiation field to the jet frame. Then, the distribution of high-energy electrons in that frame is obtained, from which the IC emission is computed. Finally, using the relativistic transformation of the radiation to the laboratory frame, one transforms the emission to that frame (for $\mu \mathrm{Q}$, see e.g. Dubus, Cerutti \& Henri 2010b; Zdziarski et al. 2012). Although this method is straightforward, in some contexts it may be more convenient to follow a different path: using Lorentz invariant quantities allows avoiding several frame transformations and provides the results in a form that illustrates the influence of different parameters clearly (see e.g. Georganopoulos et al. 2001). The difference between these two approaches is sketched in Fig. 1.

To describe the properties of non-thermal particles in the jet, we use the distribution function in the phase space:

$\mathrm{d} N=f(t, \boldsymbol{r}, \boldsymbol{p}) d^{3} \boldsymbol{r} d^{3} \boldsymbol{p}$.

We follow a general notation policy in which the uppercase ' $N$ ' refers to number of particles, i.e. a dimensionless quantity, and the lowercase letters, e.g. ' $n$ ', ' $f$ ', to densities or (differential) distributions, i.e. $\mathrm{d} N=n \mathrm{~d} X$, where $X$ is some quantity or a set of quantities.

We assume that non-thermal particles are confined in a narrow jet. Thus, in a coordinate system in which the jet is directed along the $x$-axis, and the system origin is at the CO location, the distribution function should depend on the $x$-coordinate only and on the particle momenta. The acceleration site is located at a distance $x=x_{0}$ from the $\mathrm{CO}$, and from there non-thermal particles are advected downstream along a relativistic jet that moves with bulk velocity $\beta$ $=V / c$ and Lorentz factor $\Gamma=1 / \sqrt{1-\beta^{2}}$ (see Fig. 2, where the model geometry is illustrated).

The acceleration site can be associated for instance with a recollimation shock and may vary with time: $x_{0}=x_{0}(t)$ (see e.g. Perucho, Bosch-Ramon \& Khangulyan 2010). However, for the sake of simplicity, it is typically assumed that this distance changes slowly compared to the characteristic advection/cooling times, which are also significantly shorter than the binary orbital period (e.g. BoschRamon \& Khangulyan 2009).

To compute the radiation accurately, it is necessary to specify the momentum distribution of the particles. The conventional assumption is that the particles are isotropic in the jet co-moving frame, and that are injected following a certain energy distribution 


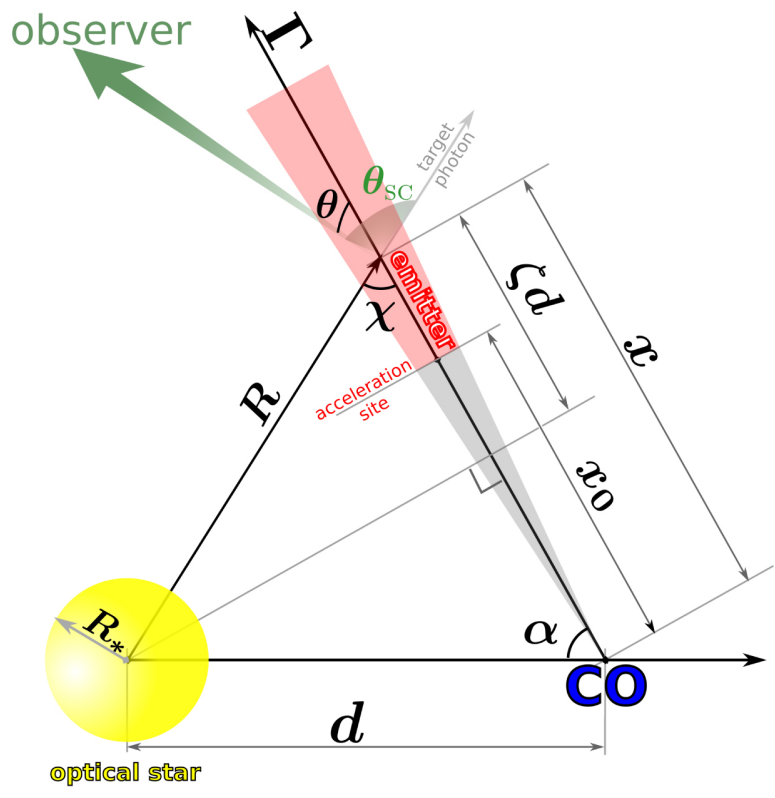

Figure 2. Sketch of the considered jet geometry.

(typically a power law) at $x_{0}^{\prime}{ }^{2}$ In the jet co-moving frame, the particle distribution function is non-stationary:

$f^{\prime}\left(t^{\prime}, \boldsymbol{r}^{\prime}, \boldsymbol{p}^{\prime}\right)=\Theta\left(x^{\prime}-x_{0}^{\prime}\left(t^{\prime}\right)\right) \delta\left(y^{\prime}\right) \delta\left(z^{\prime}\right) g^{\prime}\left(p^{\prime}, \tau\right)$,

where $x_{0}^{\prime}=x_{0} / \Gamma-\beta c t^{\prime}$ is the location of the acceleration site in the co-moving frame, $\Theta$ the Heaviside function, $\delta$ the Dirac $\delta$-function, and $\tau$ the proper age of the particle population. As nonthermal particles are accelerated at $x_{0}^{\prime}$, one obtains

$\tau\left(t^{\prime}, x^{\prime}\right)=t^{\prime}-\frac{x_{0}-\Gamma x^{\prime}}{\Gamma \beta c}$.

The evolution of the high-energy particles, which is determined by $g^{\prime}$, depends also on the parameters that define the jet orientation and $x_{0}$, which for simplicity are assumed to be constant.

The internal energy of the plasma per volume unit can be obtained as

$e\left(t^{\prime}, \boldsymbol{r}^{\prime}\right)=\Theta\left(x^{\prime}-x_{0}^{\prime}\left(t^{\prime}\right)\right) \delta\left(y^{\prime}\right) \delta\left(z^{\prime}\right) \int \mathrm{d}^{3} \boldsymbol{p}^{\prime} \varepsilon^{\prime} g^{\prime}\left(p^{\prime}, \tau\right)$,

and the energy injected ${ }^{3}$ in non-thermal particles at $x=x_{0}$ is

$L_{\mathrm{NT}}=\Gamma^{2} c \beta \int \mathrm{d}^{3} \boldsymbol{p}^{\prime} \varepsilon^{\prime} g^{\prime}\left(p^{\prime}, 0\right)$.

The up-scattering rate of target photons to gamma-rays is determined by the differential cross-section:

$\frac{\mathrm{d} \sigma}{\mathrm{d} E_{\gamma}}=\frac{\mathrm{d} \sigma}{\mathrm{d} E_{\gamma}}\left(\varepsilon, E_{\gamma}, \varepsilon_{\mathrm{ph}}, \theta_{\mathrm{SC}}\right)$,

where $\varepsilon=\sqrt{m_{\mathrm{e}}^{2} c^{4}+p^{2} c^{2}}$ is the electron energy, with $m_{\mathrm{e}}$ being the electron mass, $E_{\gamma}$ the gamma-ray energy, $\varepsilon_{\mathrm{ph}}$ the target photon energy, and $\theta_{\mathrm{SC}}$ the scattering angle, i.e. the angle between the target photon momentum and the observer direction (Aharonian \&

\footnotetext{
${ }^{2}$ The quantities with primes refer to the jet co-moving frame.

${ }^{3}$ To remain consistent with the standard one-zone modelling, here one ignores the pressure of non-thermal particles; accounting for their pressure would increase the required injected luminosity by a $\approx 30$ per cent, for the same $g$.
}

Atoyan 1981). The scattered photons move in the direction of the electron with accuracy $\sim m_{\mathrm{e}} c^{2} / \varepsilon(\ll 1$ in the relativistic regime), thus an observer located in the direction $\boldsymbol{n}_{0}$ should detect emission produced by particles with $\boldsymbol{p}=\boldsymbol{p} \boldsymbol{n}_{0}$.

In the laboratory frame, the gamma-ray spectrum can be obtained from

$$
\frac{\mathrm{d} N_{\gamma}}{\mathrm{d} t \mathrm{~d} E_{\gamma} \mathrm{d} \Omega}=\int c\left(1-\cos \theta_{\mathrm{SC}}\right) \frac{\mathrm{d} \sigma}{\mathrm{d} E_{\gamma}} f\left(\tilde{t}, \boldsymbol{r}, \boldsymbol{p} \boldsymbol{n}_{0}\right) p^{2} \mathrm{~d} n_{\mathrm{ph}} \mathrm{d} p \mathrm{~d} V
$$

where $\theta_{\text {sc }}$ in equation (7) depends on $\boldsymbol{r}$, the location of the source of target photons, and also the orbital phase, the latter playing an important role in shaping the gamma-ray light curve.

Photons emitted at $\tilde{t}(\boldsymbol{r})=T+\frac{r n_{0}}{c}$ ( $T$ is the time at which a hypothetical photon at $\boldsymbol{r}=0$ would be produced) will be simultaneously detected by the observer. The emission will arrive to the observer at a time $t=T+T_{\mathrm{PROP}}$, where $T_{\mathrm{PROP}}$ is the time required for the hypothetical photon to travel to the observer. The linear relation between $t$ and $T$ implies that equation (7) describes both emitted and received photons, which is not always the case (see e.g. Rybicki \& Lightman 1979).

The phase-space distribution function is a Lorentz invariant, $f(t$, $\boldsymbol{r}, \boldsymbol{p})=f^{\prime}\left(t^{\prime}, \boldsymbol{r}^{\prime}, \boldsymbol{p}^{\prime}\right)$, where $t^{\prime}, \boldsymbol{r}^{\prime}$, and $\boldsymbol{p}^{\prime}$ are related to $t, \boldsymbol{r}$, and $\boldsymbol{p}$, respectively, by Lorentz transformations (e.g. Landau \& Lifshitz 1975). For $\tilde{t}^{\prime}$ and $\tilde{\tau}$, one obtains

$\tilde{t}^{\prime}(\tilde{t}, x)=\Gamma T+\frac{x}{(c \beta)} \frac{\mathcal{D}-\Gamma}{\mathcal{D} \Gamma}$,

and

$\tilde{\tau}=\tau\left(\tilde{t}^{\prime}, x^{\prime}(\tilde{t}, x)\right)=\frac{x-x_{0}}{\beta c \Gamma}$,

where $\mathcal{D}=(\Gamma(1-\beta \cos \theta))^{-1}$ is the Doppler boosting factor, with $\theta$ being the angle between the jet velocity and the observer direction. The argument of the Heaviside function transforms simply as

$\Theta\left(x^{\prime}-x_{0}^{\prime}\left(t^{\prime}\right)\right)=\Theta\left(\frac{x-x_{0}}{\Gamma}\right)=\Theta\left(x-x_{0}\right)$.

Thus, one obtains that the distribution function is stationary in the laboratory frame:

$f(t, \boldsymbol{r}, \boldsymbol{p})=\Theta\left(x-x_{0}\right) \delta(y) \delta(z) g^{\prime}\left(p^{\prime}, \frac{x-x_{0}}{\beta c \Gamma}\right)$,

but it is anisotropic because of the $p_{x}$ dependence in $p^{\prime}=$ $\sqrt{\Gamma\left(\varepsilon / c-\beta p_{x}\right)-m_{\mathrm{e}}^{2} c^{2}}$.

The integral over $\mathrm{d} y \mathrm{~d} z$ can be computed yielding

$\frac{\mathrm{d} N_{\gamma}}{\mathrm{d} t \mathrm{~d} E_{\gamma} \mathrm{d} \Omega}=\int_{x_{0}}^{\infty} c\left(1-\cos \theta_{\mathrm{SC}}\right) \frac{\mathrm{d} \sigma}{\mathrm{d} E_{\gamma}} \frac{\mathrm{d} N_{e}}{\mathrm{~d} \varepsilon \mathrm{d} x} \mathrm{~d} \varepsilon \mathrm{d} n_{\mathrm{ph}} \mathrm{d} x$,

where

$\frac{\mathrm{d} N_{e}(\varepsilon, x)}{\mathrm{d} \varepsilon \mathrm{d} x}=g^{\prime}\left(\frac{p}{\mathcal{D}}, \frac{x-x_{0}}{\beta c \Gamma}\right) \frac{p^{2}}{c}$

is the effective energy distribution density ${ }^{4}$ of electrons emitting towards the observer.

For the derivation of equations (12) and (13), one considered that the injected particle distribution is relativistic, $\varepsilon \simeq c p \gg \mathcal{D} m_{\mathrm{e}} c^{2}$.

${ }^{4}$ We distinguish between energy distribution, i.e. $\mathrm{d} N=n_{1} \mathrm{~d} \varepsilon$, and energy distribution density, i.e. $\mathrm{d} N=n_{2} \mathrm{~d} \varepsilon \mathrm{d} V$ or $\mathrm{d} N=n_{3} \mathrm{~d} \varepsilon \mathrm{d} x$. 
Since the target density and scattering angle change along the jet to obtain the IC emission, it is necessary to know the distribution of the particles along the jet.

\section{EVOLUTION OF NON-THERMAL PARTICLES}

\subsection{General case}

Since in the jet co-moving frame particles are isotropic, it is convenient to use the energy distribution density:

$\mathrm{d} N=n^{\prime}\left(\varepsilon^{\prime}\right) \mathrm{d} \varepsilon^{\prime} \mathrm{d} V^{\prime}=4 \pi p^{\prime 2} f^{\prime} \mathrm{d} p^{\prime} \mathrm{d} V^{\prime}$.

Using the energy-momentum relation, $\varepsilon^{\prime 2}=c^{2} p^{\prime 2}+m_{\mathrm{e}}^{2} c^{4}$, one can substitute energy as $\mathrm{d} \varepsilon^{\prime}=c^{2} p^{\prime} \mathrm{d} p^{\prime} / \varepsilon^{\prime}$. Thus, one obtains $n^{\prime}=$ $\left(4 \pi / c^{2}\right) \varepsilon^{\prime} p^{\prime} f^{\prime}$. For $x^{\prime}>x_{0}^{\prime}$, the energy dependence of the $g$ function can be obtained as

$g^{\prime}\left(p^{\prime}, \tau\right)=\int_{\text {jet }} \mathrm{d} y^{\prime} \mathrm{d} z^{\prime} f^{\prime} \propto \frac{c^{2} n^{\prime}\left(\varepsilon^{\prime}\right)}{4 \pi \varepsilon^{\prime} p^{\prime}}$.

For the sake of simplicity, we normalize the particle energy density with equation (5), i.e. with a condition that directly determines $g^{\prime}$.

Under the continuous-loss approximation, particle evolution is described as

$n^{\prime}\left(\varepsilon^{\prime}\right) \mathrm{d} \varepsilon^{\prime} \mathrm{d} V^{\prime}=n^{\prime}\left(\varepsilon_{0}^{\prime}\right) \mathrm{d} \varepsilon_{0}^{\prime} \mathrm{d} V_{0}^{\prime}$.

The energy $\varepsilon_{0}^{\prime}$ in equation (16) evolves accordingly to the particle cooling equation:

$\frac{\mathrm{d} \varepsilon_{0}^{\prime}}{\mathrm{d} \tau}=\dot{\varepsilon}\left(\varepsilon_{0}^{\prime}, \tau\right)$

with the initial condition $\varepsilon_{0}^{\prime}(\tilde{\tau})=\varepsilon^{\prime}$. The non-thermal energy loss term typically accounts for synchrotron, IC, and adiabatic losses of electrons:

$\dot{\varepsilon}\left(\varepsilon_{0}^{\prime}, \tau\right)=\dot{\varepsilon}_{\mathrm{IC}}\left(\varepsilon_{0}^{\prime}, \tau\right)+\dot{\varepsilon}_{\mathrm{SYN}}\left(\varepsilon_{0}^{\prime}, \tau\right)+\dot{\varepsilon}_{\mathrm{AD}}\left(\varepsilon_{0}^{\prime}, \tau\right)$

in the jet co-moving frame. If the particle distribution function is defined according to equation (2), the volume element occupied by the non-thermal particles can be taken as constant $\mathrm{d} V^{\prime}=\mathrm{d} V_{0}^{\prime}$ for a constant-velocity jet (this remains correct independent of the jet geometry - see the discussion after equation 29). Thus, one obtains

$g \propto \frac{c^{2} n_{0}^{\prime}\left(\varepsilon_{0}^{\prime}\right)}{4 \pi \varepsilon^{\prime} p^{\prime}} \frac{\mathrm{d} \varepsilon_{0}^{\prime}}{\mathrm{d} \varepsilon^{\prime}}$.

If the injection spectrum is a power law in energy with index $\alpha_{\mathrm{INJ}}$, then in the ultrarelativistic limit one obtains

$g^{\prime}\left(p^{\prime}, \tau\right)=\frac{A^{\prime} c^{2} \varepsilon_{0}^{\prime-\alpha_{\mathbb{N} J}}}{4 \pi p^{\prime} \varepsilon^{\prime}} \frac{\mathrm{d} \varepsilon_{0}^{\prime}}{\mathrm{d} \varepsilon^{\prime}}$,

where $\varepsilon_{0}^{\prime}$ corresponds to $\varepsilon_{0}^{\prime}=\varepsilon_{0}^{\prime}(0)$. The normalization constant $A^{\prime}$ is determined by equation (5). Then, the energy distribution density, equation (13), can be represented as

$\frac{\mathrm{d} N_{e}(\varepsilon, x)}{\mathrm{d} \varepsilon \mathrm{d} x}=\frac{A^{\prime} \mathcal{D}^{2} \varepsilon_{0}^{\prime-\alpha_{\mathrm{INJ}}}}{4 \pi} \frac{\mathrm{d} \varepsilon_{0}^{\prime}}{\mathrm{d} \varepsilon^{\prime}}$

for $\varepsilon^{\prime}=\varepsilon / \mathcal{D}$, assuming the relativistic regime, $\varepsilon^{\prime} \approx p^{\prime} c$.

\subsection{Compact emitter}

When particles are advected along the jet, the intensity of the magnetic and the photon field, and the rate of adiabatic losses, can change, meaning that the above equation does not have an analytic solution in the general case. If advection is slow compared to radiative cooling, equation (18) becomes simpler since the loss rates can be considered steady. In this case, the formal solution of equation (17) is

$$
\int_{\varepsilon_{0}^{\prime}}^{\varepsilon^{\prime}} \frac{\mathrm{d} \hat{\varepsilon}}{\dot{\varepsilon}(\hat{\varepsilon})}=\tilde{\tau}
$$

which should be considered an algebraic equation that determines $\varepsilon_{0}^{\prime}$ as a function of $\varepsilon^{\prime}$ and $\tilde{\tau}$. Once the original electron energy is obtained, one can express the ratio of the infinitesimal energy intervals as

$\frac{\mathrm{d} \varepsilon_{0}^{\prime}}{\mathrm{d} \varepsilon^{\prime}}=\frac{\dot{\varepsilon}\left(\varepsilon_{0}^{\prime}\right)}{\dot{\varepsilon}\left(\varepsilon^{\prime}\right)}$.

\subsection{Synchrotron-Thomson losses; extended emitter}

The cooling rate for electrons interacting with background photons in the Klein-Nishina regime has a rather complicated dependence on electron energy, which makes finding an analytic solution for equation (17) difficult. In contrast, if the IC cooling proceeds in the Thomson regime, the energy loss term has a simple dependence on energy, which is identical to that of synchrotron cooling.

One can find an analytic solution if the dominant radiative cooling process is IC in the Thomson regime, or synchrotron emission. In this case, radiation losses have the following dependence on energy:

$\dot{\varepsilon}_{\mathrm{IC}}+\dot{\varepsilon}_{\mathrm{SYN}}=-a \varepsilon^{\prime 2}$,

where $a$ does not depend on electron energy. The coefficient $a$ accounts for the synchrotron and IC losses and can vary within the jet, i.e. can be a function of $\tau$ :

$$
\begin{aligned}
a & =a_{\mathrm{IC}}+a_{\mathrm{SYN}}, \\
a_{\mathrm{IC}} & =\frac{4}{3} \frac{\sigma_{\mathrm{T}} c}{\left(m_{\mathrm{e}} c^{2}\right)^{2}} w_{\mathrm{ph}}^{\prime}, \\
a_{\mathrm{SYN}} & =\frac{4}{3} \frac{\sigma_{\mathrm{T}} c}{\left(m_{\mathrm{e}} c^{2}\right)^{2}} w_{\mathrm{B}}^{\prime},
\end{aligned}
$$

where $\sigma_{\mathrm{T}}$ is the Thomson cross-section; $w_{\mathrm{ph}}^{\prime}$ and $w_{\mathrm{B}}^{\prime}$ are the energy densities of the target photons and the magnetic field in the jet frame, respectively.

If the dominant photon field is provided by the companion star, then

$w_{\mathrm{ph}}^{\prime}=\mathcal{D}_{*}^{-2} \frac{L_{*}}{4 \pi R^{2} c}$,

where $L_{*}$ and $R$ are the luminosity of the star and the distance to it, respectively, and the factor

$\mathcal{D}_{*}=\frac{1}{\Gamma(1-\beta \cos \chi)}$

accounts for the transformation of the photon field to the jet comoving frame (see e.g. Zdziarski et al. 2012; Khangulyan, Aharonian \& Kelner 2014), where $\chi$ is the angle between the jet bulk velocity and the target photon momentum in the laboratory frame (see Fig. 2, where the model geometry is illustrated).

The energy density of the magnetic field is

$w_{\mathrm{B}}^{\prime}=\frac{B^{\prime 2}}{8 \pi}$, 
where $B^{\prime}$ is the strength of the magnetic field in the jet co-moving frame in $\mathrm{G}$.

The rate of adiabatic losses is

$\dot{\varepsilon}_{\mathrm{AD}}\left(\varepsilon_{0}^{\prime}\right)=\frac{1}{3} \frac{\mathrm{d} \ln \rho}{\mathrm{d} \tau} \varepsilon_{0}^{\prime}$,

where $\rho$ is the plasma density in the flow co-moving frame. We note that $\rho$ corresponds to the jet material. The contribution of the non-thermal particles described by equation (2) to this density might be very small. The shape of the jet (e.g. cylindrical or conical) determines the rate of adiabatic losses. The factor $\delta(y) \delta(z)$ in equation (11) does not imply any limitation on the jet shape. The meaning of this factor is that the conditions for the non-thermal particles do not change considerably across the jet, and equations (2) and (11) describe the properties of the particle distribution integrated over the jet cross-section.

Equation (18) can be written as

$\frac{\mathrm{d}}{\mathrm{d} \tau}\left(\frac{\rho^{1 / 3}}{\varepsilon_{0}^{\prime}}\right)=\rho^{1 / 3}(\tau) a(\tau)$,

which provides a relation between particle energy at the injection and the emission points as

$$
\begin{aligned}
\left(\frac{\rho^{1 / 3}(\tilde{\tau})}{\varepsilon^{\prime}}\right)-\left(\frac{\rho_{0}^{1 / 3}}{\varepsilon_{0}^{\prime}}\right) & =\int_{0}^{\tilde{\tau}} \rho^{1 / 3}\left(t^{\prime}\right) a\left(t^{\prime}\right) \mathrm{d} t^{\prime} \\
& =\int_{x_{0}}^{x} \rho^{1 / 3}(\hat{x}) a(\hat{x}) \frac{\mathrm{d} \hat{x}}{\Gamma \beta c},
\end{aligned}
$$

where the relation between $\tilde{\tau}$ and $x$ comes from equation (9). Since the RHS of equation (31) does not depend on energy, the ratio of the infinitesimal energy intervals is

$\frac{\mathrm{d} \varepsilon_{0}^{\prime}}{\mathrm{d} \varepsilon^{\prime}}=\left(\frac{\varepsilon_{0}^{\prime}}{\varepsilon^{\prime}}\right)^{2}\left(\frac{\rho(\tilde{\tau})}{\rho_{0}}\right)^{1 / 3}$

where $\rho_{0}$ and $\varepsilon_{0}^{\prime}$ are the initial plasma density and particle energy, respectively. The initial energy is

$$
\varepsilon_{0}^{\prime}=\frac{\varepsilon^{\prime}\left(\frac{\rho_{0}}{\rho(\tilde{\tau})}\right)^{1 / 3}}{1-\varepsilon^{\prime} \int_{0}^{\tilde{\tau}} a\left(t^{\prime}\right)\left(\frac{\rho\left(t^{\prime}\right)}{\rho(\tilde{\tau})}\right)^{1 / 3} \mathrm{~d} t^{\prime}} .
$$

For a power-law injection spectrum, equation (32) allows us to obtain the electron energy distribution density with equation (21). For a non-power-law injection, one should use equation (19), or equivalently equation (15) with the following particle energy distribution:

$$
n^{\prime}\left(\varepsilon^{\prime}\right)=\frac{n_{0}^{\prime}\left(\varepsilon_{0}^{\prime}\right)\left(\frac{\rho_{0}}{\rho(\tilde{\tau})}\right)^{1 / 3}}{\left(1-\varepsilon^{\prime} \int_{0}^{\tilde{\tau}} a\left(t^{\prime}\right)\left(\frac{\rho\left(t^{\prime}\right)}{\rho(\tilde{\tau})}\right)^{1 / 3} \mathrm{~d} t^{\prime}\right)^{2}},
$$

where $n_{0}^{\prime}$ is proportional to the injection spectrum.

\subsection{Synchrotron-Thomson losses; compact emitter}

If the rate of radiative losses remains constant over the cooling distance, one can take the parameter $a$ as a constant. However, the plasma density dependence should be preserved in this equation, as otherwise a constant density would imply to ignore adiabatic losses completely. The density evolution is determined by the structure of the jet. For example, for a steady conical jet the mass conservation yields

$x^{2} \rho \Gamma \beta=$ const.
The evolution of the macroscopic quantities in jets is a subject for dedicated (magneto)hydrodynamic simulations (see e.g. Perucho et al. 2010) and is beyond the scope of this paper. Equation (35) does not allow determining the density of the jet material since the jet may undergo bulk acceleration. For the sake of simplicity, we assume that the jet velocity remains constant in the region relevant for gamma-ray production. In this case, $\rho$ decreases as $\propto x^{-2}$ and the integral term in equation (34) is

$$
\begin{aligned}
& \varepsilon^{\prime} a \int_{x_{0}}^{x}\left(\frac{x^{\prime}}{x}\right)^{-2 / 3} \frac{\mathrm{d} x^{\prime}}{\Gamma \beta c}=\frac{3 a \varepsilon^{\prime}}{\Gamma \beta c}\left(x-x_{0}\left(\frac{x}{x_{0}}\right)^{2 / 3}\right) \\
& =3 a \varepsilon^{\prime}\left(\tilde{\tau}+\frac{x_{0}}{\Gamma \beta c}\left(1-\left(1+\frac{\tilde{\tau} \Gamma \beta c}{x_{0}}\right)^{2 / 3}\right)\right) .
\end{aligned}
$$

For a short cooling distance compared to $x_{0}$, this reduces to

$\varepsilon^{\prime} a \int_{x_{0}}^{x}\left(\frac{x^{\prime}}{x}\right)^{-2 / 3} \frac{\mathrm{d} x^{\prime}}{\Gamma \beta c} \approx a \varepsilon^{\prime} \tilde{\tau}$,

which coincides with a solution without adiabatic losses. This calculation illustrates, to some extent, a trivial physical fact: In steady jets, the adiabatic losses might be important only in EEs.

As shown above, assuming a compact emitter (CE) in a steady jet implies a small impact of adiabatic losses. Adopting a power law in energy with index $\alpha_{\mathrm{INJ}}$ for the injected particles, one obtains for a CE

$n^{\prime}\left(\varepsilon^{\prime}\right) \propto\left(1-a \tilde{\tau} \varepsilon^{\prime}\right)^{\alpha_{\mathrm{INJ}}-2} \varepsilon^{\prime-\alpha_{\mathrm{INJ}}}$.

Accordingly with equation (20), one obtains that

$g^{\prime}\left(\frac{p}{\mathcal{D}}, \frac{x-x_{0}}{\beta c \Gamma}\right)=\frac{A^{\prime} \mathcal{D}^{2} c}{4 \pi p^{2}}\left(1-a \frac{x-x_{0}}{\beta c \Gamma} \frac{p c}{\mathcal{D}}\right)^{\alpha_{\mathrm{IN} J}-2}\left(\frac{c p}{\mathcal{D}}\right)^{-\alpha_{\mathrm{IN}}}$,

in the limit of $\varepsilon \simeq c p \gg \mathcal{D} m_{\mathrm{e}} c^{2}$. The maximum energy in equation (39), $\varepsilon_{\text {MAX }}^{\prime}$, is limited by the injection process, and the following relation should be fulfilled: $\varepsilon^{\prime} /\left(1-a \tilde{\tau} \varepsilon^{\prime}\right)<\varepsilon_{\mathrm{MAX}}^{\prime}$. In equation (12), the limit imposed by the maximum energy translates into the integral upper limit:

$x_{\mathrm{MAX}}=x_{0}+\frac{c \beta \Gamma}{a} \frac{\varepsilon_{\mathrm{MAX}}^{\prime}-\varepsilon^{\prime}}{\varepsilon_{\mathrm{MAX}}^{\prime} \varepsilon^{\prime}}$.

The dominance of radiation cooling over advection implies that the cooling length, $\left(x_{\operatorname{MAx}}-x_{0}\right)$, is small compared to the characteristic distance over which the loss rate can change significantly. Under these conditions, as noted, one can compute the integral over $x$ analytically:

$$
\begin{aligned}
& \int_{x_{0}}^{x_{\mathrm{MAX}}} \mathrm{d} x\left(1-a \frac{x-x_{0}}{\beta \Gamma} \frac{p}{\mathcal{D}}\right)^{\alpha_{\mathrm{INJ}}-2} \\
& =\frac{\beta \mathcal{D} \Gamma}{a p\left(\alpha_{\mathrm{INJ}}-1\right)}\left[1-\left(1-u_{\mathrm{MAX}}\right)^{\alpha_{\mathrm{INJ}}-1}\right] \simeq \frac{\beta \mathcal{D} \Gamma}{a p\left(\alpha_{\mathrm{INJ}}-1\right)},
\end{aligned}
$$

where $u_{\mathrm{MAX}}=\left(\varepsilon_{\mathrm{MAX}}^{\prime}-c p / \mathcal{D}\right) / \varepsilon_{\mathrm{MAX}}^{\prime} \simeq 1$, for a large maximum injection energy.

\section{INVERSE COMPTON EMISSION FROM A COMPACT RELATIVISTIC EMITTER}

Combining equations (12), (13), (39), and (41), one obtains the following expression for the spectrum produced in a compact gamma- 
ray emitter in the case of dominant Thomson or synchrotron losses:

$$
\begin{aligned}
\frac{\mathrm{d} N_{\gamma}}{\mathrm{d} t d E_{\gamma} d \Omega} \simeq & A^{\prime} \int c\left(1-\cos \theta_{\mathrm{SC}}\right) \frac{\mathrm{d} \sigma}{\mathrm{d} E_{\gamma}} \frac{\beta \mathcal{D} \Gamma}{a p\left(\alpha_{\mathrm{INJ}}-1\right)} \frac{\mathcal{D}^{2} c}{4 \pi p^{2}} \\
& \times\left(\frac{c p}{\mathcal{D}}\right)^{-\alpha_{\mathrm{INJ}}} p^{2} \mathrm{~d} p \mathrm{~d} n_{\mathrm{ph}} .
\end{aligned}
$$

According to equation (5), for a power-law injection with $\alpha_{\mathrm{INJ}}>2$, the normalization coefficient $A^{\prime}$ is approximately

$A^{\prime} \approx \frac{L_{\mathrm{NT}}\left(\alpha_{\mathrm{INJ}}-2\right)}{\Gamma^{2} \beta} \varepsilon_{\mathrm{MIN}}^{\alpha_{\mathrm{NJ}}-2}$,

yielding

$$
\begin{aligned}
\frac{\mathrm{d} N_{\gamma}}{\mathrm{d} t \mathrm{~d} E_{\gamma} \mathrm{d} \Omega}= & \frac{L_{\mathrm{NT}} \varepsilon_{\mathrm{MIN}}^{\alpha_{\mathrm{IN}}-2} c}{4 \pi a} \frac{\alpha_{\mathrm{INJ}}-2}{\alpha_{\mathrm{INJ}}-1} \frac{\mathcal{D}^{3+\alpha_{\mathrm{INJ}}}}{\Gamma} \\
& \times \int c\left(1-\cos \theta_{\mathrm{SC}}\right) \frac{\mathrm{d} \sigma}{\mathrm{d} E_{\gamma}} \varepsilon^{-\left(\alpha_{\mathrm{INJ}}+1\right)} \mathrm{d} \varepsilon \mathrm{d} n_{\mathrm{ph}} .
\end{aligned}
$$

The relativistic limit, $\varepsilon \approx p c$, was used above for the sake of simplicity.

If IC scattering of stellar photons is the dominant cooling channel, $a \approx a_{\mathrm{IC}}$, where $a_{\mathrm{IC}}$ is defined by equations (25) and (26), one obtains

$$
\frac{\mathrm{d} N_{\gamma}}{\mathrm{d} t \mathrm{~d} E_{\gamma} \mathrm{d} \Omega}=\left[\frac{\mathcal{D}^{2 \alpha_{\gamma}+1} \mathcal{D}_{*}^{2}}{\Gamma}\right] \int c\left(1-\cos \theta_{\mathrm{SC}}\right) \frac{\mathrm{d} \sigma}{\mathrm{d} E_{\gamma}} \frac{\mathrm{d} \tilde{N}}{\mathrm{~d} \varepsilon} \mathrm{d} \varepsilon \mathrm{d} n_{\mathrm{BB}},
$$

where $n_{\mathrm{BB}}=\left(2 R / R_{*}\right)^{2} n_{\mathrm{ph}}$ is the Planck distribution, $R_{*}$ the radius of the optical star, and the function $\tilde{N}$ depends on the basic parameters characterizing the system (stellar temperature $T_{*}$ and available non-thermal power), and the acceleration mechanism (accelerated particle energy dependence and minimum energy):

$$
\frac{\mathrm{d} \tilde{N}}{\mathrm{~d} \varepsilon}=\frac{3}{64 \pi} \frac{L_{\mathrm{NT}}}{\sigma_{\mathrm{B}} \sigma_{\mathrm{T}} T_{*}^{4} m_{e} c^{2}}\left(\frac{\varepsilon_{\mathrm{MIN}}}{m_{\mathrm{e}} c^{2}}\right)^{-3} \frac{\alpha_{\mathrm{INJ}}-2}{\alpha_{\mathrm{INJ}}-1}\left(\frac{\varepsilon}{\varepsilon_{\mathrm{MIN}}}\right)^{-\left(\alpha_{\mathrm{INJ}}+1\right)},
$$

where $\sigma_{\mathrm{B}}$ is the Stefan-Boltzmann constant, and the gamma-ray photon index is related to $\alpha_{\mathrm{INJ}}$ through $\alpha_{\mathrm{INJ}}=2\left(\alpha_{\gamma}-1\right)$.

If the injection process remains steady over a time similar to, or longer than, the orbital period, there are two factors that affect the variability of the GeV gamma-ray emission. The changing scattering angle and the relativistic effects can vary with the orbital phase. The relativistic effects are accounted by the term $\left[\mathcal{D}^{2 \alpha_{\gamma}+1} \mathcal{D}_{*}^{2} \Gamma^{-1}\right]$, which includes both Doppler boosting of the emission, and the transformation of the stellar photon field to the jet RF.

In line with equation (13), one can also consider the effective energy distribution of particles in the whole jet for a $\mathrm{CE}$ :

$$
\begin{aligned}
\frac{\mathrm{d} N_{e}^{(\mathrm{CE})}}{\mathrm{d} \varepsilon}= & \int_{x_{0}}^{\infty} \mathrm{d} x \frac{\mathrm{d} N_{e}(\varepsilon, x)}{\mathrm{d} \varepsilon \mathrm{d} x} \\
= & {\left[\frac{\mathcal{D}^{2 \alpha_{\gamma}+1} \mathcal{D}_{*}^{2}}{\Gamma}\right] \frac{3}{4} \frac{L_{\mathrm{NT}} R^{2}}{L_{*} \sigma_{\mathrm{T}} m_{\mathrm{e}} c^{2}}\left(\frac{\varepsilon_{\mathrm{MIN}}}{m_{\mathrm{e}} c^{2}}\right)^{-3} \frac{\alpha_{\mathrm{INJ}}-2}{\alpha_{\mathrm{INJ}}-1} } \\
& \times\left(\frac{\varepsilon}{\varepsilon_{\mathrm{MIN}}}\right)^{-\left(\alpha_{\mathrm{INJ}}+1\right)} .
\end{aligned}
$$

The emission spectrum can be written as

$\frac{\mathrm{d} N_{\gamma}}{\mathrm{d} t \mathrm{~d} E_{\gamma} \mathrm{d} \Omega}=\int c\left(1-\cos \theta_{\mathrm{SC}}\right) \frac{\mathrm{d} \sigma}{\mathrm{d} E_{\gamma}} \frac{\mathrm{d} N_{\mathrm{e}}^{(\mathrm{CE})}}{\mathrm{d} \varepsilon} \mathrm{d} \varepsilon \mathrm{d} n_{\mathrm{ph}}$, where the target photon density contains the dilution factor:

$n_{\mathrm{ph}}=\left(\frac{R_{*}}{2 R}\right)^{2} n_{\mathrm{BB}}$.

To model the GeV emission from Cygnus X-3, Dubus et al. (2010a) introduced a factor related to relativistic effects obtained for a blob emitter. Zdziarski et al. (2012) argued that this enhancement factor is not applicable to jet sources and proposed instead the factor derived by Sikora et al. (1997) for the enhancement of the emission in blazars. The impact of stellar field relativistic boosting, $\mathcal{D}_{*}$, is also accounted in the model considered by Zdziarski et al. (2012). In that study, this factor affects several different parameters: electron density; scattering angle; and scattering rate. However, combining their equations (15), (22), and (A9) and taking into account that $x$ (in the notation of Zdziarski et al. 2012) is determined by the scattering angle in the jet frame $\left(x=\mathcal{D D}_{*}(1-\cos \theta)\right.$, where $\theta$ is the scattering angle in the laboratory frame), one can derive that the IC flux is indeed $\propto \mathcal{D}_{*}^{2}$, which agrees with equations (47) and (48).

For a power-law energy distribution of electrons, the gammaray spectrum in the Thompson regime allows a simple analytic approximation (Dubus et al. 2010a; Zdziarski et al. 2012). This allows us to obtain the dependence of the IC flux analytically (for the exact expression see Zdziarski et al. 2012):

$\frac{\mathrm{d} N_{\gamma}}{\mathrm{d} t \mathrm{~d} E_{\gamma} \mathrm{d} \Omega} \propto\left(1-\cos \theta_{\mathrm{SC}}\right)^{\frac{\alpha_{\mathrm{IN}}+2}{2}} E_{\gamma}^{-\frac{\alpha_{\mathrm{INJ}}+2}{2}}$.

\section{EVOLUTION OF NON-THERMAL ELECTRONS IN AN EXTENDED EMITTER}

Equation (45) describes the IC emission if the cooling length is short compared to $x_{0}$ (see equation 40 ) and the scattering proceeds in the Thomson regime. If $\varepsilon$ is the energy responsible for the generation of gamma-rays in the laboratory frame, then combining equations (25) and (40) one obtains the condition for a compact production site:

$\frac{c \beta \Gamma \mathcal{D D}_{*}^{2}}{a_{0} x_{0} \varepsilon}\left(\frac{R}{d}\right)^{2} \ll 1$,

with

$a_{0}=\frac{4}{3} \frac{\sigma_{\mathrm{T}}}{\left(m_{\mathrm{e}} c^{2}\right)^{2}} \frac{L_{*}}{4 \pi d^{2}} \simeq \frac{L_{39}}{6 d_{12}^{2}} \mathrm{GeV}^{-1} \mathrm{~s}^{-1}$,

where $d$ is the separation between the normal star and the $\mathrm{CO}$, and fiducial parameter values between those of the gamma-ray emitting $\mu \mathrm{Q}$ Cygnus X-3 and Cygnus X-1 were used: $L_{*}=10^{39} L_{39} \mathrm{erg} \mathrm{s}^{-1}$ and $d=10^{12} d_{12} \mathrm{~cm}$. At typical Fermi LAT energies, say $E_{\gamma} \sim$ $200 \mathrm{MeV}$, the dominant contribution is produced by electron energies of a few $\mathrm{GeV}(\varepsilon=\hat{\varepsilon} \mathrm{GeV})$. Thus, the condition for the applicability of the $\mathrm{CE}$ approximation becomes

$0.2 \frac{\beta \Gamma \mathcal{D} \mathcal{D}_{*}^{2} d_{12}}{\hat{\varepsilon} L_{39}}\left(\frac{x_{0}}{d}\right)^{-1}\left(\frac{R}{d}\right)^{2} \ll 1$,

and can be violated even for a mildly relativistic jet with $\Gamma \sim 2$.

It is worth noting that for higher energy electrons, IC scattering proceeds in the Klein-Nishina regime. This should lead to an even larger extension of the production site since IC losses in the KleinNishina regime proceed slower than in Thomson.

Losses through IC are not necessarily the dominant cooling mechanism. As indicated above, synchrotron and adiabatic cooling can be also considered. Since adiabatic losses trace a change in the density, they are to be included when the CE approximation fails. 
If synchrotron losses dominate, the condition for a $\mathrm{CE}$ is determined by the magnetic field in the jet. The synchrotron time is

$t_{\mathrm{SYN}}^{\prime} \simeq 4 \times 10^{5}\left(\frac{B^{\prime}}{\mathrm{G}}\right)^{-2}\left(\frac{\hat{\varepsilon}}{\mathcal{D}}\right)^{-1} \mathrm{~s}$.

The cooling length, $x_{\mathrm{SYN}}=\Gamma \beta c t_{\mathrm{SYN}}^{\prime}$, remains small compared to $x_{0}$ if the magnetic field is

$B^{\prime} \gg 100 \sqrt{\frac{\beta \Gamma \mathcal{D}}{\hat{\varepsilon} x_{0,12}}} \mathrm{G}$.

For the sake of simplicity, let us parametrize the jet radius as a fraction of $x_{0}: r_{\mathrm{j}}=\varpi x_{0}$, where $\varpi \ll 1$. Then the Poynting energy flux in such a jet is

$$
\begin{aligned}
S & =\frac{V B^{\prime 2} \Gamma^{2} \pi^{2} x_{0}^{2}}{4 \pi} \\
& \gg 2.5 \times 10^{37} \frac{\varpi^{2} \beta^{2} \mathcal{D} \Gamma^{3}}{\hat{\varepsilon}}\left(\frac{x_{0}}{10^{12} \mathrm{~cm}}\right)^{3 / 2} \operatorname{erg~s}^{-1} .
\end{aligned}
$$

The numerical coefficient $2.5 \times 10^{37}$ corresponds to approximately 5 per cent of the Eddington luminosity for a $5 \mathrm{M}_{\odot}$ black hole. Although such a strong magnetic field cannot be excluded in Galactic jet sources, modelling tends to favour a weaker magnetization of the jet (Dubus et al. 2010a; Zdziarski et al. 2012, 2018). Thus, we conclude that there are no robust arguments excluding a significant extension of the gamma-ray production region in gamma-ray emitting $\mu \mathrm{Q}$.

\subsection{Synchrotron and adiabatic loss treatment}

The structure of equation (31) allows us to consider IC losses and synchrotron losses independently. First, we will start with synchrotron losses. The combined impact of synchrotron and adiabatic losses is determined by the following integral:

$$
\int_{0}^{\tilde{\tau}} \rho^{1 / 3}\left(t^{\prime}\right) a\left(t^{\prime}\right) \mathrm{d} t^{\prime} \propto \int_{x_{0}}^{x} \rho^{1 / 3}(\hat{x}) B^{\prime 2}(\hat{x}) \mathrm{d} \hat{x} .
$$

The density and the magnetic field depend on the structure of the jet, but most likely they can be approximated by power-law functions in a limited section of the jet. Thus, the above integral can be solved analytically yielding a description of the joint impact of synchrotron and adiabatic losses. For example, if the impact of IC losses is small, then such a treatment of adiabatic and synchrotron losses allows us to obtain a solution for equation (31), and thus to obtain an analytical description of the spatial-energy distribution of electrons in the jet.

\subsection{Thomson and adiabatic loss treatment}

For the case of dominant IC losses, the function $a$ has a more complicated structure since it depends not only on the distance from the $\mathrm{CO}$, but also on the angle between the jet velocity and target photon momenta. Modelling by Dubus et al. (2010a) suggests that the jet in Cygnus X-3 is not perpendicular to the orbital plane. Thus, we consider here also a case when the jet is inclined by a fixed angle $\alpha$. In this case, the cooling rate depends on the parameter $\zeta$ $=(x / d-\cos \alpha)$, where $\alpha$ is the angle between the jet velocity and the direction from the $\mathrm{CO}$ to the companion star (see Fig. 2, where the model geometry is illustrated). Including $\zeta$, equation (17) describing particle cooling becomes

$$
\frac{\mathrm{d}}{\mathrm{d} \zeta} \frac{\rho^{1 / 3}}{\tilde{\varepsilon}}=\rho^{1 / 3}(x(\zeta)) \frac{\zeta^{2}\left(1+\beta^{2}\right)+\sin ^{2} \alpha-2 \beta \zeta \sqrt{\zeta^{2}+\sin ^{2} \alpha}}{\left(\zeta^{2}+\sin ^{2} \alpha\right)^{2}},
$$

where the dimentionless energy, $\tilde{\varepsilon}$, is defined as

$\tilde{\varepsilon}=\varepsilon_{0}^{\prime} \frac{L_{*} \Gamma \sigma_{\mathrm{T}}}{3 \pi c \beta d\left(m_{e} c^{2}\right)^{2}}$.

In general, equation (58) does not allow an analytic solution. We can still treat it numerically, for which we will adopt a density profile of $\rho=\rho_{0}\left(x_{0} / x\right)^{2}$.

\subsection{Thomson loss treatment}

In case adiabatic losses are weak, i.e. $\rho$ is roughly constant, equation (58) determines $\tilde{\varepsilon}(x)$ :

$\frac{1}{\tilde{\varepsilon}}=\frac{\pi / 2-\chi}{\sin \alpha}\left(1+\frac{\beta^{2}}{2}\right)+\frac{2 \beta d}{R}-\frac{\beta^{2} d \sqrt{R^{2}-d^{2} \sin ^{2} \alpha}}{2 R^{2}}+\mathrm{C}$,

where $\chi=\chi(x)$ and $R=R(x)$ are functions of $x$, and $\mathrm{C}$ is an integration constant. This relation allows one to link the particle energy at the injection point $x_{0}$ to that at the emission point $x$ :

$\frac{1}{\tilde{\varepsilon}_{0}}+E\left(x_{0}\right)=\frac{1}{\tilde{\varepsilon}}+E(x)$,

where

$$
\begin{aligned}
E(x)= & -\frac{\frac{\pi}{2}-\chi(x)}{\sin \alpha}\left(1+\frac{\beta^{2}}{2}\right)-\frac{2 \beta d}{R(x)} \\
& +\frac{\beta^{2} d \sqrt{R(x)^{2}-d^{2} \sin ^{2} \alpha}}{2 R(x)^{2}} .
\end{aligned}
$$

Equation (61), together with equation (16), allows us to obtain an analytic representation of the energy distribution of the particles in the jet:

$n^{\prime}\left(\varepsilon^{\prime}\right)=\frac{\varepsilon_{0}^{\prime 2}}{\varepsilon^{\prime 2}} n_{0}^{\prime}\left(\varepsilon_{0}^{\prime}\right)$,

where the initial particle energy

$\varepsilon_{0}^{\prime}=\frac{\varepsilon^{\prime}}{1-\varepsilon^{\prime} \frac{L_{*} \Gamma \sigma_{\mathrm{T}}\left(E\left(x_{0}\right)-E(x)\right)}{3 \pi c \beta d\left(m_{\mathrm{e}} c^{2}\right)^{2}}}$

should remain smaller than the maximum energy, $\varepsilon_{\mathrm{MAX}}^{\prime}$, in the injection spectrum $n_{0}^{\prime}$.

For a power-law injection spectrum, one obtains that the distribution function is

$$
\begin{aligned}
g^{\prime}\left(\frac{p}{\mathcal{D}}, x\right)= & \frac{A^{\prime} \mathcal{D}^{2} c}{4 \pi p^{2}}\left(1-\frac{L_{*} \Gamma \sigma_{\mathrm{T}}\left(E\left(x_{0}\right)-E(x)\right)}{3 \pi \beta d\left(m_{\mathrm{e}} c^{2}\right)^{2}} \frac{p}{\mathcal{D}}\right)^{\alpha_{\mathbb{I N J}-2}} \\
& \times\left(\frac{c p}{\mathcal{D}}\right)^{-\alpha_{\mathbb{I N J}}} .
\end{aligned}
$$

Equation (65) describes the Thomson cooling of high-energy electrons in the jet also in the case when electrons may travel distances comparable to the orbital separation. This analytic solution does not account for the impact of adiabatic losses, as it is the case for instance in a cylindrical jet. Equation (65) can be easily generalized to account for synchrotron losses, for example, if the magnetic field strength has a power-law dependence.

For the computation of the IC emission, one should note that in equation (12) the photon density $n_{\mathrm{ph}}$ and the IC scattering angle 
depend on $x$. In the next section, we apply a numerical approach to compute the radiation from such an extended region.

\section{RADIATION FROM AN EXTENDED EMITTER}

To study the impact of advection in the jet, we adopt parameters similar to those of Cygnus X-3, which is a highly compact system with a very bright star, and also one of the most powerful $\mathrm{GeV}$ sources in the Galaxy. The temperature and luminosity of the donor star were adopted to be $T_{*}=10^{5} \mathrm{~K}$ and $L_{*}=1.8 \times 10^{39} \mathrm{erg} \mathrm{s}^{-1}$, respectively, and the $\mathrm{CO}$ was assumed to be located at a distance of $d=2.7 \times 10^{11} \mathrm{~cm}$ from the companion star. This separation distance is the largest allowed by Koljonen \& Maccarone (2017) and corresponds to a $\mathrm{CO}$ with $M_{\mathrm{CO}} \simeq 5 \mathrm{M}_{\odot}$ and a WR star with $M_{\mathrm{WR}} \simeq 15 \mathrm{M}_{\odot}$.

\subsection{Emitter size}

First, we study the size of the gamma-ray emitting region for different locations of the acceleration site and for different orientations of the jet. We adopt the maximum energy in the injection spectrum to be $\varepsilon_{\mathrm{MAX}}^{\prime}=10 \mathrm{GeV}$ and compute the maximum distance that electrons with energy $\varepsilon^{\prime}$ can reach. For electrons with larger energy, $\geq 10 \mathrm{GeV}$, and the stellar temperature of Cygnus X-3, the Klein-Nishina effect should weaken the IC losses compared to the Thomson case. This should lead to an increase in the advection distance, although the impact on the GeV light curve should be small given the steepness of the measured spectrum. Efficient advection of high-energy electrons may have an important influence on the multi-GeV gamma-ray flux, since in this energy band the gammagamma attenuation is strong, and more efficient advection of the emitting electrons can significantly reduce the attenuation factor.

The advection distance, $x_{\mathrm{MAX}}$, is determined through equation (58) as

$$
\begin{aligned}
& \frac{\rho_{0}^{1 / 3}}{\tilde{\varepsilon}_{\mathrm{MAX}}}-\frac{\rho^{1 / 3}\left(x_{\mathrm{MAX}}\right)}{\tilde{\varepsilon}} \\
& =\int_{\zeta\left(x_{0}\right)}^{\zeta\left(x_{\mathrm{MAX}}\right)} \mathrm{d} \zeta \rho^{1 / 3}(x(\zeta)) \frac{\zeta^{2}\left(1+\beta^{2}\right)+\sin ^{2} \alpha-2 \beta \zeta \sqrt{\zeta^{2}+\sin ^{2} \alpha}}{\left(\zeta^{2}+\sin ^{2} \alpha\right)^{2}},
\end{aligned}
$$

which in the case of weak adiabatic losses reduces to

$$
\frac{1}{\tilde{\varepsilon}_{\mathrm{MAX}}}+E\left(x_{0}\right)=\frac{1}{\tilde{\varepsilon}}+E\left(x_{\mathrm{MAX}}\right) \text {. }
$$

We solve numerically equation (66) for a conical jet and several jet velocities and orientations. However, if the jet expands slower than a conical one, the rate of adiabatic losses will be smaller. Thus, we also compute the case for dominant IC losses (equation 67). Fig. 3 shows the relative size of the emitting region

$\lambda=\frac{x_{\mathrm{MAX}}(\varepsilon)-x_{0}}{x_{0}}$,

for different injection points: $x_{0}=0.3 d, d$, and $3 d$ (from bottom to top); jet inclinations: $\alpha=\pi / 4, \pi / 2$, and $3 \pi / 4$ (from left to right); and jet velocities: $\beta=0.5,0.7$, and 0.9 (line colour). It is seen that even in such a compact system as Cygnus X-3, the advection of $\mathrm{GeV}$ electrons might be significant if the acceleration site is located at $d \sim 10^{12} \mathrm{~cm}$ from the $\mathrm{CO}$, in a jet that moves with a bulk Lorentz factor $\Gamma \geq 2(\beta \geq 0.87)$.

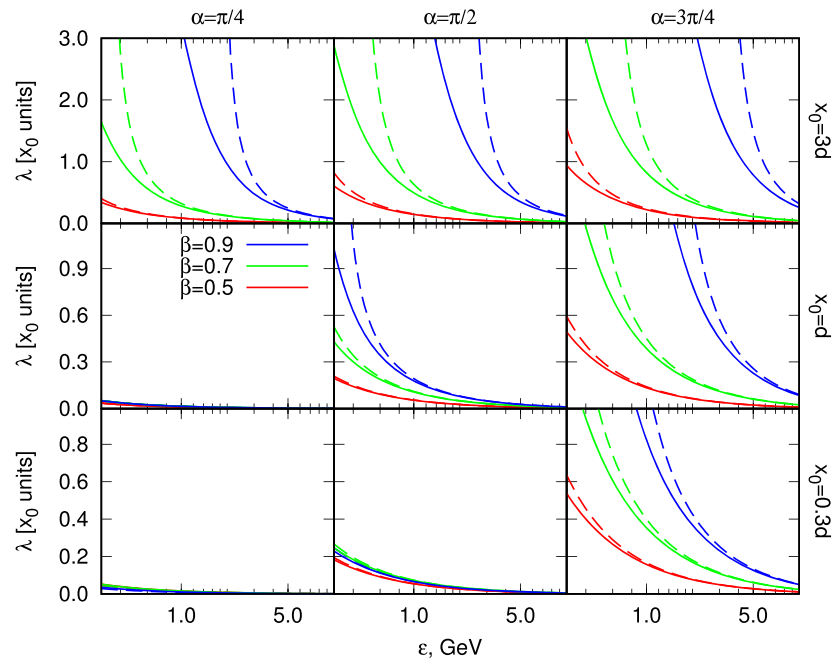

Figure 3. Relative size of the jet filled with electrons of different energies. Calculations are performed for conditions similar to Cygnus $\mathrm{X}-3$, three different jet orientations (columns for $\alpha=\pi / 4, \pi / 2$, and $\pi / 4$, injection locations (raws: $x_{0}=0.3 d, d$, and $3 d$ ), and jet velocities (colours: $\beta=0.5$, red; $\beta=0.7$, green; and $\beta=0.9$, blue). The case with negligible adiabatic losses is shown with the dashed lines and for a conical jet with the solid lines.

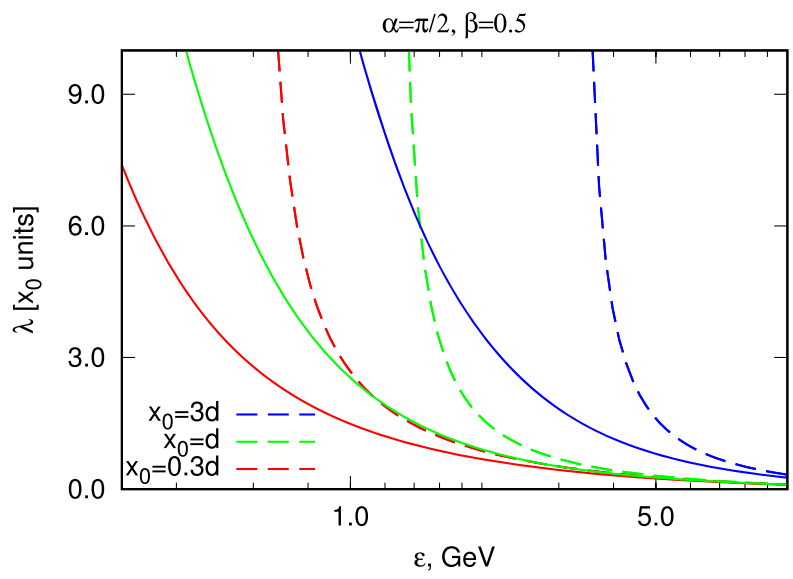

Figure 4. Relative size of the jet filled with electrons of different energies. Calculations are performed for conditions similar to those in Cygnus X-1, assuming a jet perpendicular to the orbital plane and a jet velocity of $\beta$ $=0.5$. Three different injection points are shown: $x_{0}=0.3 d$ (red), $x_{0}=$ $d$ (green), and $x_{0}=3 d$ (blue). The case with negligible adiabatic losses is shown with the dashed lines, and adiabatic losses for a conical jet with the solid lines.

For a system with a larger star separation than Cygnus X-3, the impact of advection should be stronger. For parameters similar to Cygnus X-1 $\left(T_{*}=3 \times 10^{4} \mathrm{~K}, L_{*}=8 \times 10^{38} \mathrm{erg} \mathrm{s}^{-1}\right.$, and $d=$ $3 \times 10^{12} \mathrm{~cm}$; see e.g. Caballero-Nieves et al. 2009, and reference therein), we show in Fig. 4 the extension of the GeV emitter for three different injection points: $x_{0}=0.3 d, d$, and $3 d$. As seen from the figure, under dominant IC losses the $\mathrm{CE}$ approximation for Cygnus $\mathrm{X}-1$ (or similar systems) is only justified if the injection occurs very close to the $\mathrm{CO}$, or if the jet is not relativistic. 


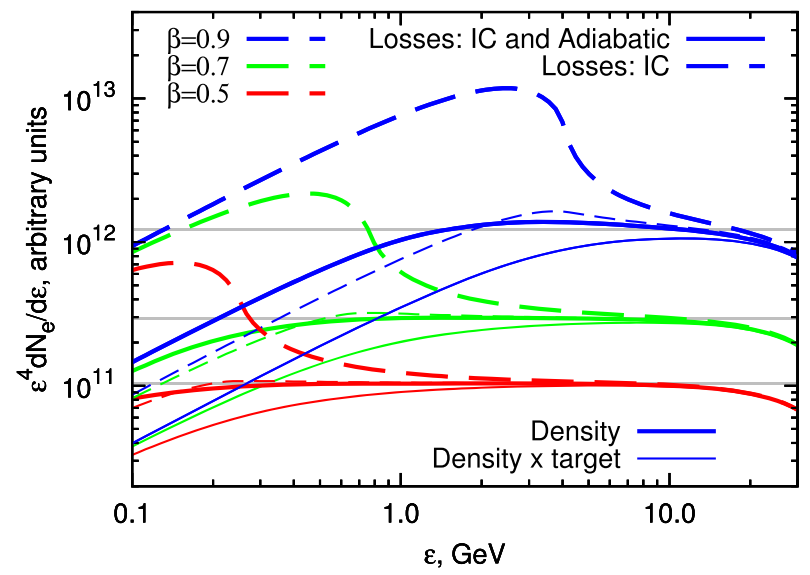

Figure 5. Energy distribution of electrons in the jet calculated for conditions similar to those in Cygnus X-3. Three different jet velocities are shown: $\beta$ $=0.5$ (red), $\beta=0.7$ (green), and $\beta=0.9$ (blue). The case with negligible adiabatic losses is shown with the dashed lines, and for a conical jet with the solid lines. The grey lines show the energy distribution obtained for a CE, equation (47). The thin lines show the electron distribution weighted with the target photon density (see equation 70 ). Other model parameters were set as $B=0, x_{0}=3 d$, and $\alpha=\pi / 2$.

\subsection{Particle distribution}

Advection affects the particle energy distribution in the jet. As the baseline case, we adopt the results obtained for a CE, equation (47). We assume that the non-thermal injection occurs at $x=3 d$, and the acceleration spectrum is steep: $\alpha_{\mathrm{INJ}}=3$. As follows from equation (50), for this electron injection spectrum the gamma-ray photon index should be $\sim 2.5$, which is roughly consistent with the spectrum detected from Cygnus X-3 with Fermi LAT (see e.g. Zdziarski et al. 2018). Thomson cooling should render an energy distribution $\propto \varepsilon^{-4}$. Thus, in Fig. 5 we plot the particle distributions multiplied by $\varepsilon^{4}$. For the case of an EE, we define the effective energy distribution in accordance with equation (13) and similar to equation (47) as

$\frac{\mathrm{d} N_{e}^{(\mathrm{EE})}(\varepsilon)}{\mathrm{d} \varepsilon}=\int_{x_{0}}^{\infty} \mathrm{d} x \frac{\mathrm{d} N_{e}(\varepsilon, x)}{\mathrm{d} \varepsilon \mathrm{d} x}$.

In Fig. 5, the results of the calculations of the electron energy distribution are shown for $\beta=0.5,0.7$, and 0.9 , and $x_{0}=3 d$. The maximum energy in the injected spectrum was assumed to be $\varepsilon_{\text {MAX }}^{\prime}=30 \mathrm{GeV}$, the Doppler boosting factor $\mathcal{D}=1.7$ (which implies different viewing angles for different jet velocities), and the magnetic field was assumed to be weak (formally set to $B=0$ ). The energy distribution of a CE, equation (47), for the same jet parameters, is shown with grey lines in Fig. 5.

As shown in Fig. 5, the energy distributions that account for advection have three characteristic features compared to equation (47). At the highest energies, there are less particles than in the CE approximation, which is caused by ignoring the injection maximum energy in equation (47) (the CE case), and thus it should not be associated with advection. At lower energies, advection leads to particle accumulation in regions with slower energy losses. Thus, for the same injection, the amount of particles for a steady jet is higher. Obviously, these features are the mostly pronounced in electron distributions computed for weak adiabatic losses. Adiabatic losses expected in conical jets appear to be significant enough to determine the shape of the electron distribution at lower energies as shown in Fig. 5 with thin lines.
To illustrate the effect of photon target dilution, we also compute the amount of emitting electrons weighted by the target photon density (the thin lines in Fig. 5):

$\frac{\mathrm{d} \bar{N}_{e}{ }^{(\mathrm{EE})}}{\mathrm{d} \varepsilon}=\int_{x_{0}}^{\infty} \mathrm{d} x \frac{\mathrm{d} N_{e}}{\mathrm{~d} \varepsilon \mathrm{d} x}\left(\frac{R_{0}}{R(x)}\right)^{2}$.

As seen in the figure, the weighted energy distribution is suppressed with respect to the CE approximation at low energies because of particle escape. In the calculations, we adopted a maximum jet length of $35 d$, as particles reaching that far from the $\mathrm{CO}$ are already strongly cooled down due to adiabatic losses. We also note that particles that reached that distance from the $\mathrm{CO}$ should not produce variable gamma-ray emission, as $\theta_{\mathrm{sc}}$ does not significantly change along the orbit. Fig. 5 shows that for $\beta \geq 0.7$, the particle energy distribution features a break or steepening at energies around $\varepsilon \simeq$ $1 \mathrm{GeV}$, which might be testable with Fermi LAT in Cygnus X-3.

\subsection{Spectral energy distribution and gamma-ray light curve}

To compute the radiative output from a jet in a binary system, it is necessary to define the inclination of the orbit and the jet orientation. The jet orientation should also include the assumption if one considers jet or counter-jet; the former is located on the same side as the observer, and the latter on the opposite side, with respect to the orbital plane. We consider two cases, $i_{\mathrm{ORB}}=30^{\circ}$ and $i_{\mathrm{ORB}}=60^{\circ}$, and the emission produced only by the jet since for relativistic bulk velocities the emission from the counter-jet is strongly suppressed. To illustrate the impact of advection, we consider the simplest case of a circular orbit, which is reasonable for Cygnus X-3. For simplicity, the jet is assumed to be perpendicular to the orbital plane. In this case, there are three relevant orbital phases: superior/inferior conjunction of the CO (SUPC/INFC); and when the $\mathrm{CO}$ crosses the plane of the sky (NODE). IC losses and radiation are affected by three angles: $\chi$ (jet velocity-photon momentum angle); $\theta$ (jet velocity-line-of-sight angle); and $\theta_{\mathrm{SC}}$ (IC scattering angle), which are shown in Fig. 2. These angles depend on the inclination of the orbit, the jet orientation, and the emitter location in the jet. The dependence of these angles on the distance from the $\mathrm{CO}$ is shown in Fig. 6 for the selected inclinations.

The combined effects of particle advection and changes in the photon density and scattering angle may affect the gamma-ray spectrum in a quite complex manner, as shown in Fig. 7. For certain orientations of the jet, the emission from the inferior conjunction is strongly suppressed by unfavourable scattering angles, in which case advection along the jet will tend to enhance the emission below $1 \mathrm{GeV}$. At high electron energies, the cooling length is shorter, and the IC cross-section has a weaker dependence on the scattering angle, so the particle transport has a minor impact on the high-energy part of the spectrum. Thus, advection may distort the spectral shape in the GeV energy band. This effect is strongly pronounced in fast jets (see top and bottom panels of Fig. 7).

Around the superior conjunction of the $\mathrm{CO}$, advection tends to harden the radiation spectrum. This is caused by the escape of lower energy electrons to regions of weaker photon fields, as illustrated in Fig. 5 with thin lines. As shown in Fig. 7, advection tends to enhance the emission around inferior conjunction and reduces the emission around superior conjunction. Thus, the orbital variation of the gamma-ray emission will be weakened by advection. This is illustrated in Fig. 8, where light curves for different jet velocities $(\beta$ $=0.7$ and $\beta=0.9)$, and inclinations $\left(i_{\mathrm{ORB}}=30^{\circ}\right.$ and $i_{\mathrm{ORB}}=60^{\circ}$ ), are shown, with the acceleration site assumed to be at $x_{0}=3 d$. It 


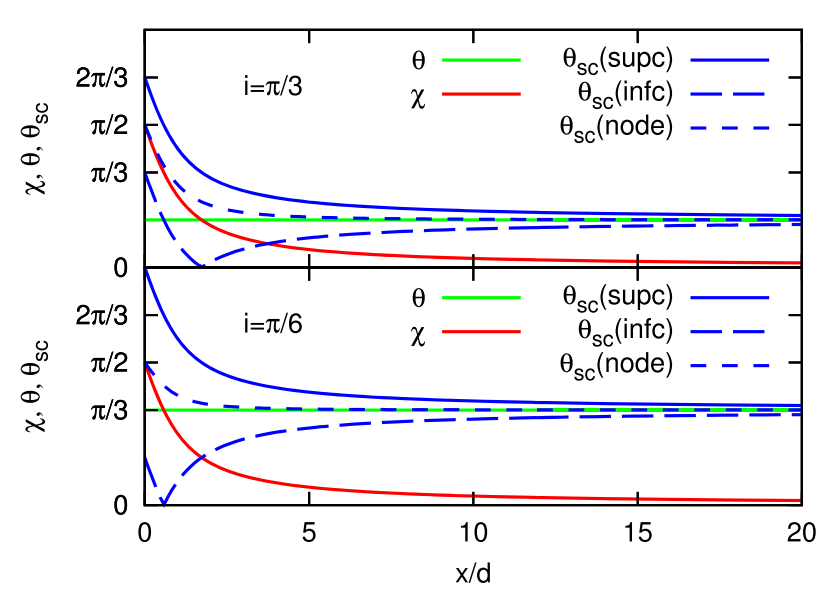

Figure 6. Characteristic angles that determine electron cooling and emission in a relativistic jet. Figure shows $\chi$ (target-photon momentum-bulk velocity), $\theta$ (observer direction-bulk velocity), and $\theta_{\mathrm{SC}}$ (observer directiontarget-photon momentum) angles. Calculations are performed for two orbital inclinations: $i_{\mathrm{ORB}}=60^{\circ}$ (top panel) and $i_{\mathrm{ORB}}=30^{\circ}$ (bottom panel). The jet was assumed to be perpendicular to the orbital plane, $\alpha=\pi / 2$, and thus only $\theta_{\mathrm{SC}}$ depends on the orbital phase; three different orbital phases are shown: SUPC (solid lines), INFC (long dashed lines), and NODE (dashed lines).

can be seen that even in the slower case with $\beta=0.7$, the orbital variation becomes significantly weaker.

As expected, adiabatic losses result in a less-extended production site, and thus the orbital phase dependence remains stronger than in the case of negligible adiabatic losses (solid versus dashed lines in Fig. 8). However, the spectral change caused by propagation effects may remain strong. In particular, the combined effects of particle advection towards regions with weaker photon field, and adiabatic cooling, result in a hardening of the photon spectrum around $0.1-1$ GeV (see Fig. 7).

\section{QUANTUM ELECTRODYNAMICS EFFECTS}

There are two quantum electrodynamics (QED) effects that may have a substantial influence on the gamma-ray emission produced in compact binary systems. The first is related to the transition from the classical Thomson limit to the quantum Klein-Nishina regime. The Thomson limit is valid when electron and target photon energies are small:

$4 \varepsilon \omega_{\mathrm{ph}}\left(1-\cos \theta_{\mathrm{SC}}\right) \ll 1$.

If the electron and the target photon energy are high enough to violate this relation, the precise QED cross-section should be used (for astrophysical conditions, see Aharonian \& Atoyan 1981). The Klein-Nishina effect has a strong impact both on the energy-loss rate and on the IC spectrum.

The second important QED effect is the gamma-gamma attenuation. Typically, in binary systems this effect is important in the $\mathrm{TeV}$ energy band (Dubus 2006). If the stellar temperature is high, as e.g. in Cygnus X-3, the attenuation might be important for gammarays with relatively low energy, $E_{\gamma} \geq 10 \mathrm{GeV}$ (Protheroe \& Stanev 1987; Moskalenko et al. 1993; Bednarek 1997; Cerutti et al. 2011; Sitarek \& Bednarek 2012). In Fig. 9, we show the attenuation factor for gamma-rays interacting with the stellar field. The target photon field is provided by the optical star with radius and temperature of $R_{*}=1.6 \times 10^{11} \mathrm{~cm}$ and $10^{5} \mathrm{~K}$, respectively. The calculation takes into account the finite size of the star integrating over the stellar surface (which can give a substantial difference compared to
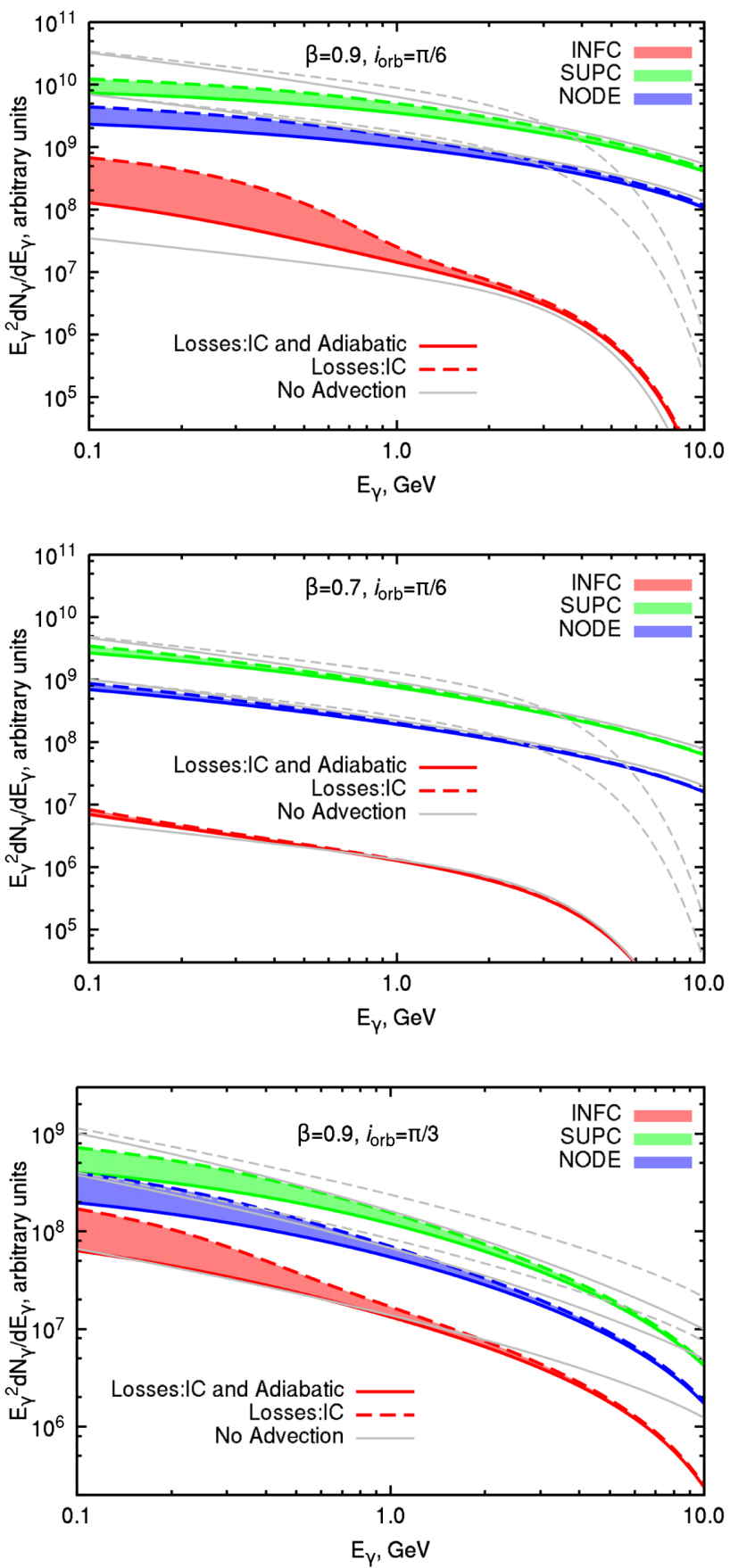

Figure 7. Spectral energy distributions of the IC emission for an EE obtained for three different orbital phases: SUPC (green); INFC (red); and NODE (blue). The jet is assumed to be perpendicular to the orbital plane, $\alpha$ $=\pi / 2$. The case with weak adiabatic losses is shown with the dashed lines, and adiabatic losses for a conical jet with the solid lines. The region between these two regimes is filled with colour. The grey lines show the spectral energy distributions obtained for a CE (equation 45). The grey dashed lines show the spectral energy distributions for SUPC and NODE phases obtained by applying the orbital-phase dependent coefficient in equation (50) to INFC spectrum obtained under CE approximation (grey solid line). Cases with $\beta$ $=0.9$ and $i_{\mathrm{ORB}}=30^{\circ}, \beta=0.7$ and $i_{\mathrm{ORB}}=30^{\circ}$, and $\beta=0.9$ and $i_{\mathrm{ORB}}=60^{\circ}$ are shown in the top, middle and bottom panels, respectively. Other model parameters were set as $B=0$ and $x_{0}=3 d$. 

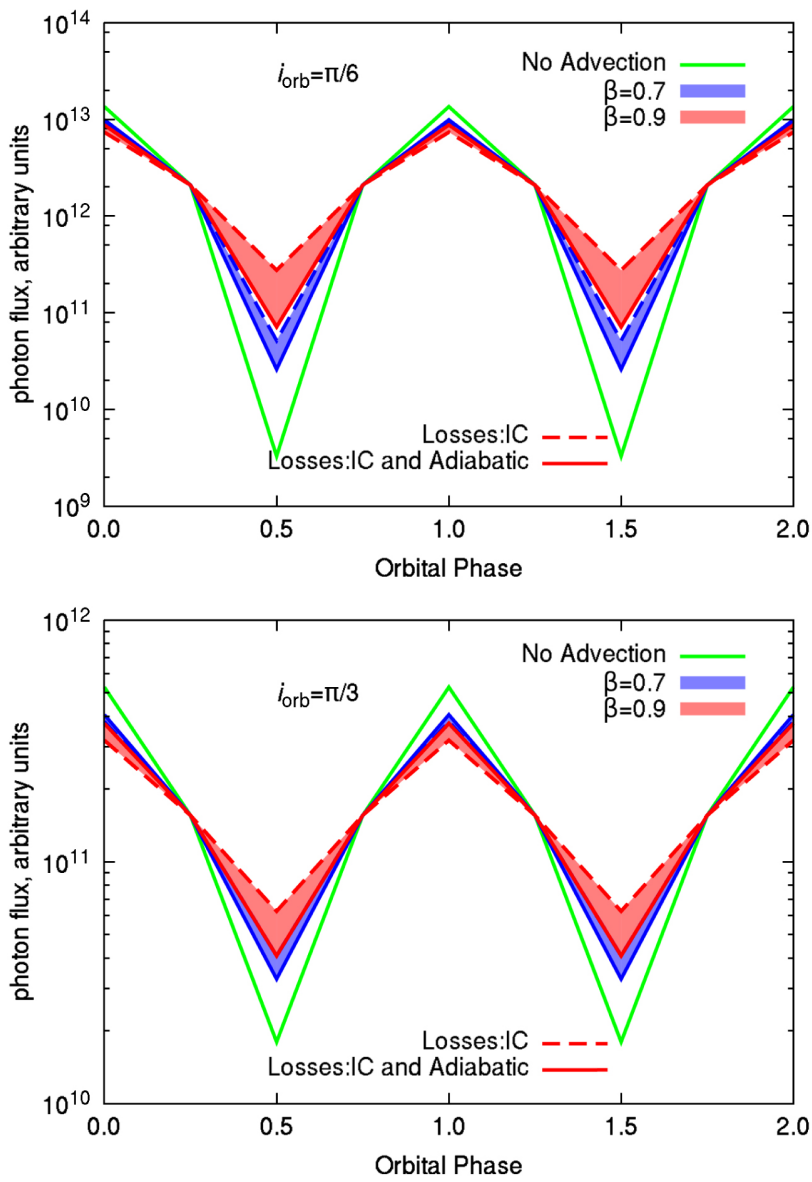

Figure 8. Light curves of the IC emission accounting for electron advection. Calculations are performed for two orbital inclinations: $i_{\mathrm{ORB}}=60^{\circ}$ (top panel) and $i_{\mathrm{ORB}}=30^{\circ}$ (bottom panel). The jet was assumed to be perpendicular to the orbital plane, $\alpha=\pi / 2$. The case with weak adiabatic losses is shown with the dashed lines, and adiabatic losses for a conical jet with the solid lines. The region between these two regimes is filled with colour. Two different jet velocities are shown with different colours: $\beta=0.7$ (blue); and $\beta=0.9$ (red). The green lines correspond to the electron spectrum obtained for a $\mathrm{CE}$ (equation 47). Other model parameters were set as $B=0$ and $x_{0}=3 d$.

calculations adopting a point-like approximation for the star if the gamma-ray emitter locates within a few stellar radius distance from the star, see e.g. Dubus 2006; Bosch-Ramon, Khangulyan \& Aharonian 2008; Bosch-Ramon \& Khangulyan 2009; Romero, Del Valle \& Orellana 2010), this accounts for the occultation by the star, as seen in the map opacity for $1 \mathrm{GeV}$ photons in Fig. 9. For $10 \mathrm{GeV}$ gamma-rays, the attenuation can be very significant for almost half of the orbit unless the gamma-ray production site is located at a large distance from the $\mathrm{CO}$. At a few $\mathrm{GeV}$, the influence of the gamma-ray absorption is smaller, although it still can suppress the emission from the counter-jet at SUPC phases, which can be relevant if the jet bulk velocity is relatively small. This may result in an additional factor affecting the orbital variability with a strong energy dependence, yielding a multi-GeV light curve significantly different from the $\mathrm{GeV}$ light curve.

In the case of Klein-Nishina losses, electrons may lose a significant fraction of energy in a single interaction, which is inconsistent with the assumptions used for the continuous-loss approximation. However, the continuous-loss approximation was shown to provide results consistent with a detailed kinetic treatment (see e.g.
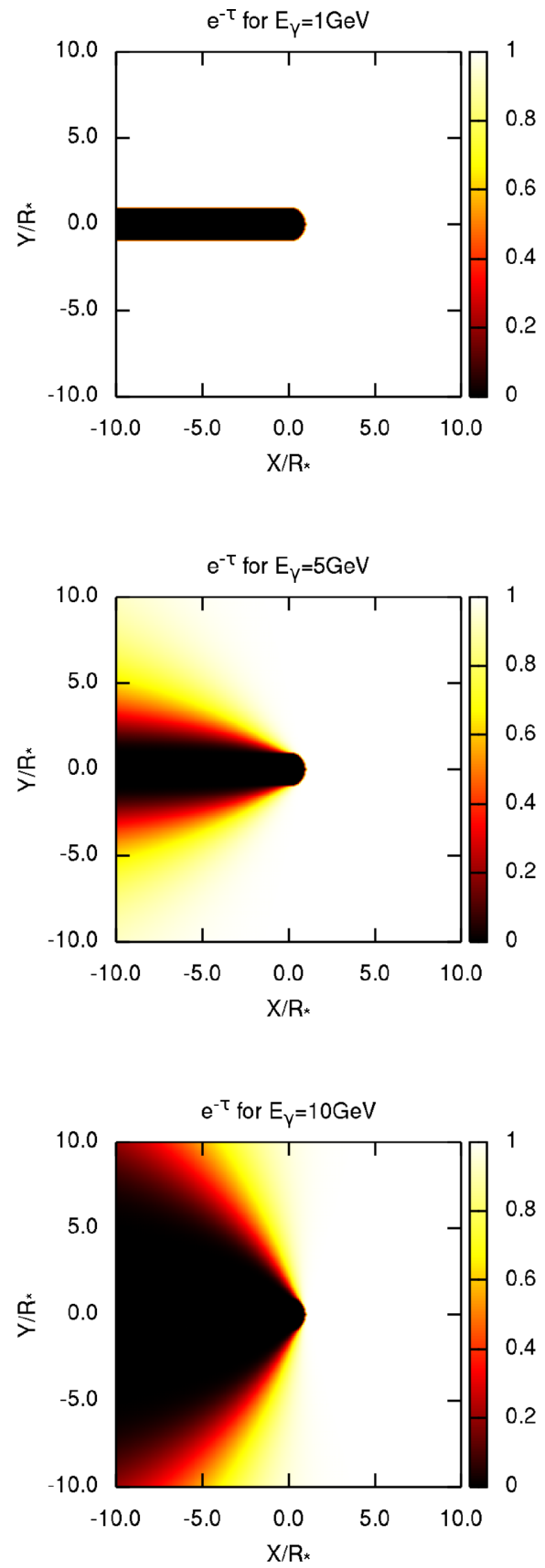

Figure 9. Gamma-gamma attenuation factor for $1 \mathrm{GeV}$ (top panel), $5 \mathrm{GeV}$ (middle panel), and $10 \mathrm{GeV}$ (bottom panel) gamma-rays traveling towards an observer looking from the right. The calculations are done for $R_{*}=$ $1.6 \times 10^{11} \mathrm{~cm}$ and $T_{*}=10^{5} \mathrm{~K}$.

Khangulyan \& Aharonian 2005). Thus, to account for the KleinNishina effect we solve equation (18) for a conical jet using the approximation for IC losses in a Planckian photon field suggested in Khangulyan et al. (2014). To compute the electron density, we use the continuous-loss approximation, i.e. the energy distribution density of electrons is described by equation (21). The influence of the reduction of energy losses due to the Klein-Nishina effect is shown in Fig. 10. It is seen that the weakening of the IC energy losses results in $\mathrm{a} \sim 30$ per cent increase of the number density of GeV electrons. 


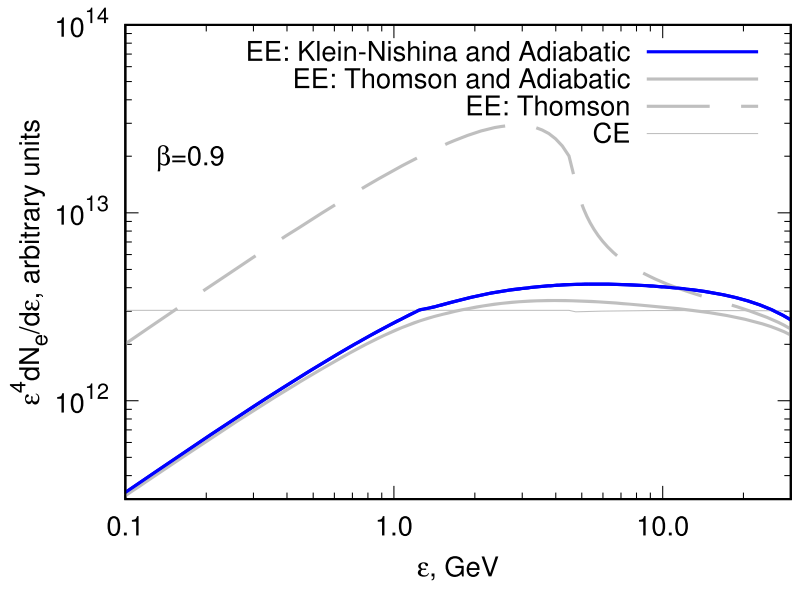

Figure 10. Energy distribution of electrons in the jet calculated for conditions similar to those in Cygnus X-3. The jet velocity was assumed to be $\beta$ $=0.9$ and non-thermal electrons with $\alpha_{\mathrm{INJ}}=3$ were injected at a distance $x$ $=3 d$ from the CO. The grey lines correspond to densities shown in Fig. 4 and obtained for Thomson losses: CE (CE, thin line); EE with Thomson losses only (dashed line); and EE with adiabatic losses for a conical jet (solid line). The solid blue line shows the energy distribution of electrons computed using an accurate IC loss prescription, under adiabatic losses in a conical jet. The jet was taken perpendicular to the orbital plane, $\alpha=\pi / 2$.

For each considered gamma-ray energy and location in the jet, we compute the gamma-gamma opacity, $\tau_{\gamma \gamma}$, in the stellar photon field in the direction of the observer. The influence of different transport and cooling assumptions is illustrated in Fig. 11. As seen in the figure, Klein-Nishina IC cooling has a similar impact at different orbital phases on the gamma-ray emission intensity, resulting in a small transformation of the light-curve shape. In contrast, gamma-gamma attenuation strongly affects the gamma-ray spectrum above $5 \mathrm{GeV}$ for SUPC. We note that Fig. 11 shows the emission produced in the jet; for the counter-jet the impact should be considerably stronger, for relatively low jet velocities. Thus, a detailed study of multi-GeV gamma-ray emission from Cygnus X-3 may significantly constrain the possible locations of the production site.

\section{CONCLUSIONS}

We have studied the properties of the gamma-ray emitting region in a relativisitic jet in a binary system. To facilitate the interpretation of the results, we have used an approach based on the distribution function in the phase space, which is Lorentz invariant. This allows obtaining results in a compact form that permits studying the influence of different parameters in a clearer way. The main focus of the study was on the impact of advection on the gamma-ray spectrum and light curve.

For the case of a compact production site, we have obtained an analytic representation of the energy distribution of the emitting electrons. When IC cooling dominates over advection, the gammaray spectrum, given by equation (45), has a simple form that allows one to determine the process that affects the variability of the emission. Namely, it contains three factors that change with orbital phase: (i) IC proceeds in the anisotropic regime, and the scattering angle varies along the orbit (Khangulyan et al. 2008; Dubus et al. 2010a; Zdziarski et al. 2012); (ii) the Doppler boosting factor, $\left[\mathcal{D}^{2 \alpha_{\gamma}+1} \Gamma^{-1}\right]$, that accounts for the relativistic transformation of radiation produced in a stationary jet (Sikora et al. 1997); and (iii)
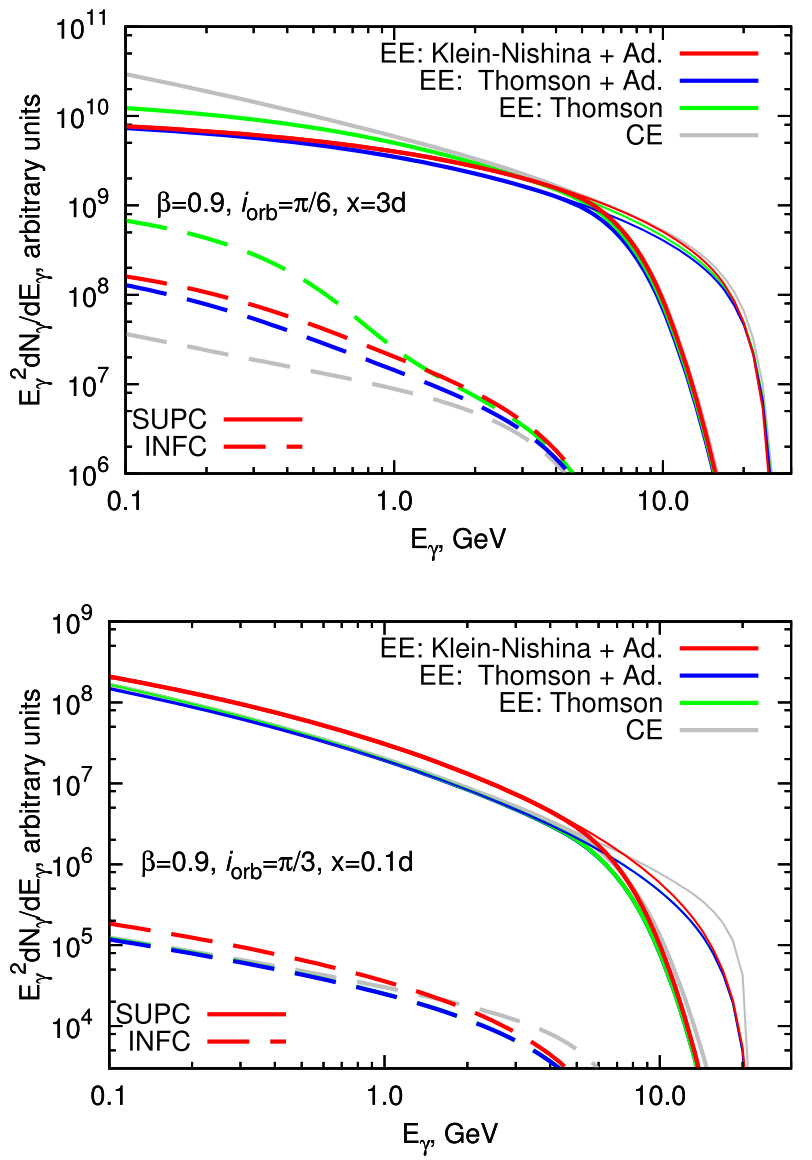

Figure 11. Comparison of gamma-ray spectral energy distributions calculated under different assumptions on transport and cooling for emission obtained for a CE (grey lines); dominant Thomson cooling (green lines); Thomson cooling with adiabatic losses in a conical jet (blue); and KleinNishina cooling with adiabatic losses in a conical jet (red lines). Two orbital phases are shown: SUPC (solid lines) and INFC (dashed lines). Calculations account for gamma-gamma attenuation in the photon field of the optical companion. For SUPC, the intrinsic spectra are shown with thin lines (for INFC the attenuation is negligible). The jet velocity was assumed to be $\beta=0.9$, and two different injection points are considered: $x=3 d$ ( $i_{\mathrm{ORB}}=\pi / 6$, top panel) and $x=0.1 d\left(i_{\mathrm{ORB}}=\pi / 3\right.$, bottom panel). The jet was taken perpendicular to the orbital plane, $\alpha=\pi / 2$.

in the case of dominant IC losses, an additional factor, $\mathcal{D}_{*}^{2}$, should be introduced. The stellar photon boosting effect on cooling can be ignored if the dominant losses are due to synchrotron cooling.

Adiabatic losses can be relevant only if relativistic particles are advected along the jet over a distance in which the jet material density undergoes a significant change. In particular, this can be the case for low-energy electrons that are subject to slower radiative losses. In the case of a (at least) mildly relativistic jet, $\Gamma \geq 2$, advection might be important for $\mathrm{GeV}$ emitting electrons even in the most compact binaries such as Cygnus X-3. In the case of dominant radiative losses, we have obtained an analytic solution that describes the properties of non-thermal electrons in a relativistic inclined jet. This solution can, however, be generalized to the case when adiabatic losses are important under weak IC losses, i.e. covering a broad range of synchrotron and adiabatic losses.

It is generally expected that in gamma-ray emitting $\mu \mathrm{Q}$ IC losses should dominate over synchrotron for $\mathrm{GeV}$ electrons (see e.g. Zdziarski et al. 2012). Thus, as test cases, we have considered 
two cases for EEs: (i) dominant IC losses, which allow an analytic solution for the particle density, equation (63); and (ii) the case with IC and adiabatic losses, the latter being expected in a conical jet, for which a numerical treatment has been applied. The simulations have shown that, in systems similar to Cygnus X-3, particle advection may have a significant impact on the gamma-ray light curve if the jet velocity is high, $\beta \geq 0.7$. For even faster jet velocities, $\beta \sim 0.9$, one should also expect a strong transformation of the gamma-ray spectrum from different orbital phases. In a more extended system, e.g. in Cygnus X-1, advection is very important unless synchrotron losses prevent efficient particle transport (see equation 55), which is probably not very realistic.

In the specific case of Cygnus X-3, the stellar companion should be very hot, $T_{*} \simeq 10^{5} \mathrm{~K}$. For such a target photon field, two QED effects may influence the electron transport and gamma-ray spectrum in the GeV energy band. The Klein-Nishana effect weakens the IC energy losses and affects the gamma-ray spectrum, and gammagamma absorption can significantly suppress the flux above a few $\mathrm{GeV}$. To study the influence of these effects, we have performed detailed calculations of the electron transport, radiation, and gammagamma opacity. Since in the case of Cygnus X-3, the orbital separation is comparable to the stellar radius, in the calculations of the gamma-gamma opacity we have accounted for the finite size of the optical star. The simulations show that the Klein-Nishina effect has a small impact on the intrinsic gamma-ray spectra. Unless the gamma-ray production site is located at large distance from the $\mathrm{CO}$, $x \gg 3 d$, the gamma-gamma attenuation should significantly affect the spectrum at multi-GeV energies, $E_{\gamma}>5 \mathrm{GeV}$.

To summarize, we have performed a detailed study of the IC process in realistic jets in compact binary systems. The performed study has revealed that the particle advection along the jet might be important even in a very compact binary system, e.g. in Cygnus X3. In systems similar to Cygnus X-1, advection should be accounted for even in the case of a weakly relativistic jet.

If adiabatic losses are weak, which would be the case, e.g. in cylindrical jets, advection can impact significantly the gamma-ray emission, potentially leading to a strong dependence of the gammaray spectrum shape on the orbital phase. For advection in a conical jet, adiabatic losses weaken the effects on the spectrum.

Independent of the dominant cooling channel, advection results in a significant weakening of the orbital phase dependence. Thus, if the properties of the accelerator in Cygnus X-3 and Cygnus X1 are similar, one should expect differences in the orbital phase dependence of the $\mathrm{GeV}$ emission between these two systems.

To illustrate the relevance of this effect, in Fig. 12, we show the light curves computed for a system similar to Cygnus X-1 (the temperature and luminosity of the optical star are taken as $T_{*}=3 \times$ $10^{4} \mathrm{~K}$ and $L_{*}=8 \times 10^{38} \mathrm{erg} \mathrm{s}^{-1}$, respectively; the CO was assumed to be in a circular orbit with $d=3.2 \times 10^{12} \mathrm{~cm}$ ).

The IC emission shown in Fig. 12 was averaged over two orbital phase bins: $|\phi|<0.25$ and $|\phi|>0.25$, the orbit being $-0.25<\phi<$ $0.75)$. The injection point was assumed to be located at $x_{0}=4 d$, and the injection spectrum and jet velocity were assumed to be $\propto \varepsilon^{-4}$ and $\beta=0.5$, respectively. The orbital inclination was selected to be $i_{\mathrm{ORB}}=\pi / 3$, and the figure includes only the contribution from the jet (i.e. the counter-jet emission is not accounted for because it is expected to be relatively small and to weaken the orbital phase dependence even stronger). The data points are from Zdziarski et al. (2017), and the open and filled squares correspond to the emission expected from a $\mathrm{CE}$ and an $\mathrm{EE}$, respectively. The adiabatic losses were assumed to be weak and the magnetic field set to $B=0$, so the dominant cooling mechanism is the Thomson scattering. As seen

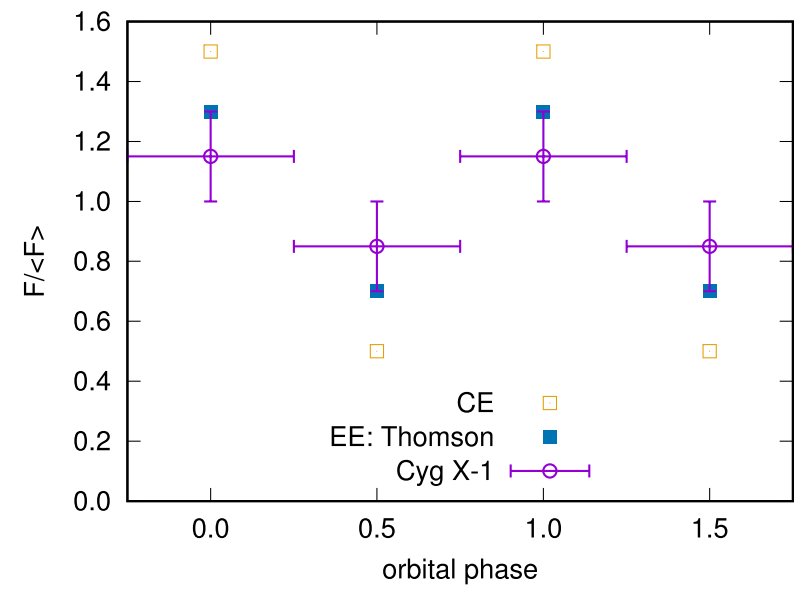

Figure 12. Light curves of the IC emission from a system similar to Cygnus $\mathrm{X}-1: T_{*}=3 \times 10^{4} \mathrm{~K}, L_{*}=8 \times 10^{38} \mathrm{erg} \mathrm{s}^{-1}, d=3.2 \times 10^{12} \mathrm{~cm}$ (circular orbit), $\beta=0.5$, and $i_{\mathrm{ORB}}=60^{\circ}$. The jet was assumed to be perpendicular to the orbital plane, $\alpha=\pi / 2$. The case with weak adiabatic losses is shown with the filled squares and the emission expected from a CE is shown with the open squares. The data points are adopted from Zdziarski et al. (2017). Other model parameters were set as $B=0$ and $x_{0}=4 d$.

from Fig. 12, the advection may provide a possible explanation for a weaker orbital phase dependence of the $\mathrm{GeV}$ emission from Cygnus X-1 and alleviate the requirement for an SSC contribution. Zdziarski et al. (2017) studied the broad-band emission and gammaray variability in Cygnus $\mathrm{X}-1$ for the parameter space with $x_{0} \ll$ $d$. In that parameter space, it was found that external Compton models, including those with an EE, are incompatible with the Fermi LAT emission from Cygnus X-1, and a better agreement can be achieved if one assumes a highly clumpy jet, which enhances the SSC emission.

In the case of a conical jet (as was assumed by Zdziarski et al. 2017), adiabatic losses lead to considerable cooling at distances $x$ $\sim x_{0}$, so plasma cools down on a scale in which the IC regime does not change for $x_{0} \ll d$. Thus, advection cannot considerably affect the IC light curve for parameters adopted by Zdziarski et al. (2017). The simulations shown in Fig. 12 show that for $x_{0} \geq d$, advection can improve the agreement between the observational data from Cygnus X-1 and predictions of models that account for stellar IC only. We note, however, that the calculations presented in Fig. 12 are for illustrative purposes only and cannot substitute a detailed broad-band study (as the one presented in Zdziarski et al. 2017).

The influence of advection on the gamma-ray light curve also significantly affects the ability of one-zone models (Dubus et al. 2010a; Zdziarski et al. 2012, 2018) to accurately infer the properties of the gamma-ray production sites even in the case of the most compact binary systems. For example, Zdziarski et al. (2018) suggested that the Fermi LAT emission in Cygnus X-3 is best explained by IC scattering from a production site located at $x=$ $2.3 d$ in a jet with $\beta=0.73$. As shown by our simulations, for this location of the production site the transport effects might be relevant.

We present in this paper the theoretical framework and discuss the impact of advection on the GeV gamma-ray spectrum and light curve. A detailed application to the gamma-ray data of Cygnus X-3 will be presented in a forthcoming paper. 


\section{ACKNOWLEDGEMENTS}

We want to thank the anonymous referee for useful and constructive comments and suggestions. This work was supported by JSPS KAKENHI Grant Number JP18H03722. VB-R acknowledges support by the Spanish Ministerio de Economía y Competitividad (MINECO/FEDER, UE) under grants AYA2016-76012-C3-1$\mathrm{P}$, with partial support by the European Regional Development Fund (ERDF/FEDER), and MDM-2014-0369 of ICCUB (Unidad de Excelencia 'María de Maeztu'), and the Catalan DEC grant 2014 SGR 86.

\section{REFERENCES}

Abdo A.A. et al., 2009, Science, 326, 1512

Aharonian F. A., Atoyan A. M., 1981, Ap\&SS, 79, 321

Bednarek W., 1997, A\&A, 322, 523

Bodaghee A., Tomsick J. A., Pottschmidt K., Rodriguez J., Wilms J., Pooley G. G., 2013, ApJ, 775, 98

Bosch-Ramon V., Khangulyan D., 2009, Int. J. Mod. Phys. D, 18, 347

Bosch-Ramon V., Khangulyan D., Aharonian F. A., 2008, A\&A, 482, 397

Bulgarelli A. et al., 2012, A\&A, 538, A63

Caballero-Nieves S. M. et al., 2009, ApJ, 701, 1895

Cerutti B., Dubus G., Malzac J., Szostek A., Belmont R., Zdziarski A. A., Henri G., 2011, A\&A, 529, A120

Corbel S. et al., 2012, MNRAS, 421, 2947

Dubus G., 2006, A\&A, 451, 9

Dubus G., Cerutti B., Henri G., 2010a, MNRAS, 404, L55

Dubus G., Cerutti B., Henri G., 2010b, A\&A, 516, A18

Georganopoulos M., Kirk J. G., Mastichiadis A., 2001, ApJ, 561, 111

Georganopoulos M., Aharonian F. A., Kirk J. G., 2002, A\&A, 388, L25

Jackson J. C., 1972, Nature (Phys. Sci.), 236, 39

Kaufman Bernadó M. M., Romero G. E., Mirabel I. F., 2002, A\&A, 385, L10

Khangulyan D., Aharonian F.A., , 2005, in Aharonian F. A., Völk H. J., Horns D., eds, AIP Conf. Proc. Vol. 745, High Energy Gamma-Ray Astronomy. American Institute of Physics, New York, p. 359

Khangulyan D., Aharonian F., Bosch-Ramon V., 2008, MNRAS, 383, 467

Khangulyan D., Aharonian F. A., Kelner S. R., 2014, ApJ, 783, 100
Koljonen K. I. I., Maccarone T. J., 2017, MNRAS, 472, 2181

Landau L. D., Lifshitz E. M., 1975, Course of Theoretical Physics-Pergamon International Library of Science, Technology, Engineering and Social Studies. Pergamon Press, Oxford

Malyshev D., Zdziarski A. A., Chernyakova M., 2013, MNRAS, 434, 2380

Martí J., Paredes J. M., Peracaula M., 2001, A\&A, 375, 476

Mioduszewski A. J., Rupen M. P., Hjellming R. M., Pooley G. G., Waltman E. B., 2001, ApJ, 553, 766

Moskalenko I. V., Karakula S., Tkaczyk W., 1993, MNRAS, 260, 681

Pepe C., Vila G. S., Romero G. E., 2015, A\&A, 584, A95

Perucho M., Bosch-Ramon V., Khangulyan D., 2010, A\&A, 512, L4

Piano G. et al., 2012, A\&A, 545, A110

Piano G., Munar-Adrover P., Verrecchia F., Tavani M., Trushkin S. A., 2017, ApJ, 839, 84

Protheroe R. J., Stanev T., 1987, ApJ, 322, 838

Romero G. E., Kaufman Bernadó M. M., Mirabel I. F., 2002, A\&A, 393, L61

Romero G. E., Del Valle M. V., Orellana M., 2010, A\&A, 518, A12

Rybicki G. B., Lightman A. P., 1979, Radiative Processes in Astrophysics. Wiley, New York

Sikora M., Madejski G., Moderski R., Poutanen J., 1997, ApJ, 484, 108

Sitarek J., Bednarek W., 2012, MNRAS, 421, 512

Tavani M. et al., 2009, Nature, 462, 620

Vila G. S., Romero G. E., 2010, MNRAS, 403, 1457

Vila G. S., Romero G. E., Casco N. A., 2012, A\&A, 538, A97

Williams P. K. G. et al., 2011, ApJ, 733, L20

Zanin R., Fernández-Barral A., de Oña Wilhelmi E., Aharonian F., Blanch O., Bosch-Ramon V., Galindo D., 2016, A\&A, 596, A55

Zdziarski A. A., Sikora M., Dubus G., Yuan F., Cerutti B., Ogorzałek A., 2012, MNRAS, 421, 2956

Zdziarski A. A., Stawarz Ł., Pjanka P., Sikora M., 2014a, MNRAS, 440, 2238

Zdziarski A. A., Pjanka P., Sikora M., Stawarz Ł., 2014b, MNRAS, 442, 3243

Zdziarski A. A., Malyshev D., Chernyakova M., Pooley G. G., 2017, MNRAS, 471, 3657

Zdziarski A. A. et al., 2018, MNRAS, 479, 4399

This paper has been typeset from a $\mathrm{T}_{\mathrm{E}} \mathrm{X} / \mathrm{L}_{\mathrm{A}} \mathrm{E} \mathrm{X}$ file prepared by the author. 